\title{
Recent developments in homobimetallic reagents and catalysts for organic synthesis
}

\author{
WU Wei, GU DeLiang, WANG ShiMeng, NING YingNan \& MAO GuoLiang* \\ Provincial Key Laboratory of Oil and Gas Chemical Technology, College of Chemistry and Chemical Engineering, Northeast Petroleum \\ University, Daqing 163318, China
}

Received September 13, 2010; accepted November 4, 2010; published online May 10, 2011

\begin{abstract}
Organometallics are a family of useful organic chemicals because they play important roles in organic synthesis as reagents and as catalysts. They can be classified according to the number of metals they contain. Bimetallic compounds are important organometallics and they are either homobimetallic or heterobimetallic depending on whether the two metals are the same or different. In this paper, we focus on homobimetallic compounds. Homobimetallic compounds are generally used as dianions to react with electrophiles in organic synthesis. Recently, homobimetallics have also been used as catalysts in organic reactions such as in asymmetric reactions.
\end{abstract}

homobimetallics, reagent, catalyst, synthesis, application

Citation: $\quad$ Wu W, Gu D L, Wang S M, et al. Recent developments in homobimetallic reagents and catalysts for organic synthesis. Chinese Sci Bull, 2011, 56: 1753-1769, doi: 10.1007/s11434-011-4470-7

\section{Homobimetallic reagents for organic synthesis}

Homobimetallic reagents can be classified according to the relative positions of the two carbon atoms that bear the metal atoms. The positions of the metals will affect their reactivity. The reactions of bimetallic compounds have been summarized previously [1]. Recently, the cyclization reactions of dianions were thoroughly reviewed by Langer et al. [2]. In this paper, we compile, analyze and discuss recent developments in homobimetallic reagent-mediated reactions. Some important results that have been summarized in previous reviews will also be included.

Among bimetallics, organodilithium compounds are a family of very important intermediates. Many other organobimetallic compounds can be obtained by the transmetalation of corresponding dilithio compounds. Organodilithium compounds can be synthesized by several methods including hydrogen-lithium exchange (deprotonation), halogen-lithi-

*Corresponding author (email: maoguoliang@ @epu.edu.cn) um exchange, transmetalation reactions, carbon-heteroatom bond cleavage and the lithiation of multiple carbon-carbon bonds, etc. [3].

\section{$1.1 \quad$ 1,1-Bimetallic compounds}

1,1-Bimetallic compounds are also referred to as geminal bimetallics. Because two metals are attached to the same carbon atom, geminal bimetallics exhibit very interesting reaction properties. Marek and Normant summarized the synthesis and reactions of both homo- and hetero-sp ${ }^{3}$ geminal organodimetallics [4]. These included dilithioalkane, dimagnesioalkane, dialuminioalkane, diborioalkane and dizincoalkane reagents.

As highly reactive dianion species geminal bimetallic compounds form open-chain products by reacting with monofunctional electrophiles followed by the addition of water. Cyclization reactions can also take place if the geminal bimetallic is treated with a dielectrophile [2].

(1) 1,1-Dilithio compounds. The lithiation of compounds containing a $\mathrm{CH}_{2}$ group at the ortho- position of a 
sulfone or a nitrile can lead to the formation of a "true $\alpha, \alpha$-dianion", which has been confirmed by single crystal crystallography [5]. These dianions can undergo cyclization reactions with biselectrophiles.

Langer and co-workers [6] carried out the first direct transformation of silyl enol ethers to the geminal lithiated allene 1 (Scheme 1), which was trapped by a ketone to give the corresponding diol $\mathbf{2}$. A subsequent domino reaction with nitriles gives the corresponding dihydropyrimidine 3 [7].

Synthetic equivalents of 1,1-dilithioethylene 4 [8] and 1,1-dilithiocyclopropane $\mathbf{5}$ [9] were prepared by sequential lithiation using two different methods. These methods provide a convenient way to introduce two different electrophiles (Scheme 2) .

(2) 1,1-Dizinco compounds. The transition metal catalyzed cross-coupling reaction of an organometallic reagent with an organic electrophile is one of the most important skeleton-constructing methods in organic synthesis. By employing a geminal bimetallic reagent, sequential coupling reactions can be carried out to introduce two electrophiles onto one carbon atom. Matsubara's group realized this using bis(iodozincio)methane $\mathbf{6}$ and 1,1-bis(iodozincio)ethane (Scheme 3) [10].

\section{$1.2 \quad$ 1,2-Bimetallic compounds}

Diphenylacetylene can be reduced by metallic lithium to form the cis-dilithium adduct $\mathbf{7}$, which was converted into cis-stilbene after treatment with methanol [11]. It has been found that trimethylsilyl substituted styrenes can also be reduced by metallic lithium to form the corresponding 1,2-dilithio intermediate 8 (Scheme 4) [12]. Yus et al. [13] successfully reduced methyl-substituted styrene with metallic lithium in the presence of a catalytic amount of 4,4'-ditert-bytylbiphenyl (DTBB). The dilithio compounds 9 formed were captured in situ by carbonyl compounds or by chlorotrimethylsilane.

A very special type of 1,2-dilithio compound originates from ortho-carborane. Many substituents can be introduced to the carborane skeleton through transmetalation or other reactions. The direct insertion of sulfur or selenium into the carbon-lithium bonds leads to corresponding ortho-carboranedithiolate and diselenolate complexes. (Pentamethylcyclopentadienyl)iridium $(\mathrm{Cp} * \mathrm{Ir})$ was introduced to the orthocarboranediselenolate dianion to form a 5-membered ring structure (compound $\mathbf{1 0}$ in Scheme 5) [14]. The interaction between orthocarboranedithiolate and diselenolate complexes with lanthanocene chlorides afforded a series of

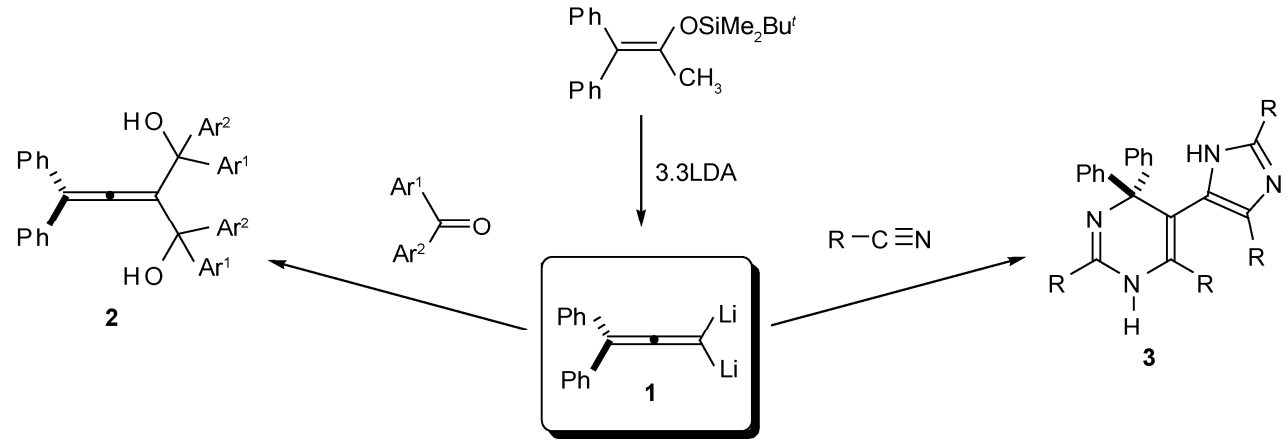

Scheme 1 Preparation of geminal lithiated allenes and their application in organic synthesis.

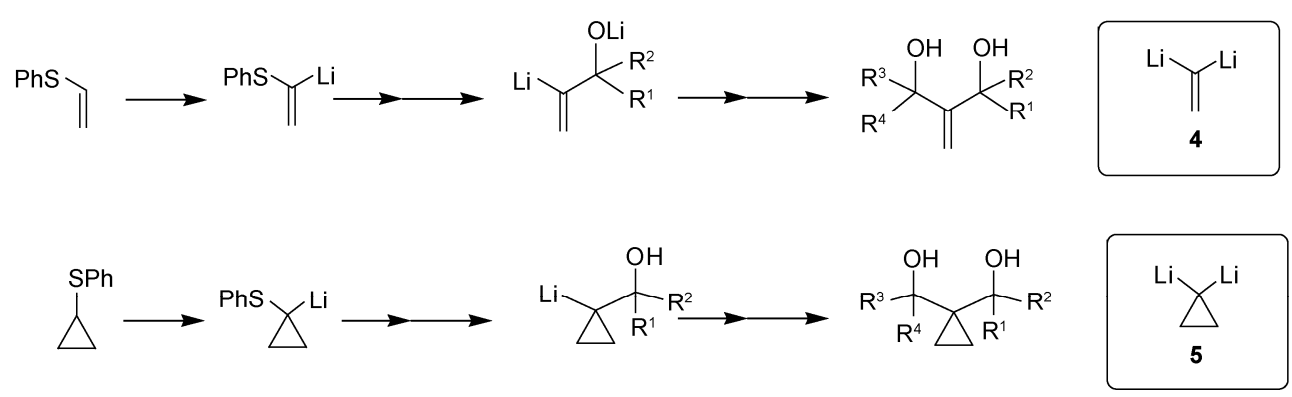

Scheme 2 Reactions of geminal dilithio equivalents.

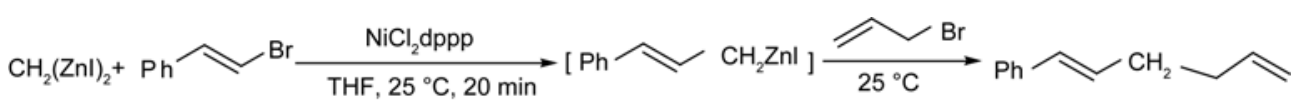

Scheme 3 Sequential coupling reaction of 1,1-bis(iodozincio)methane. 

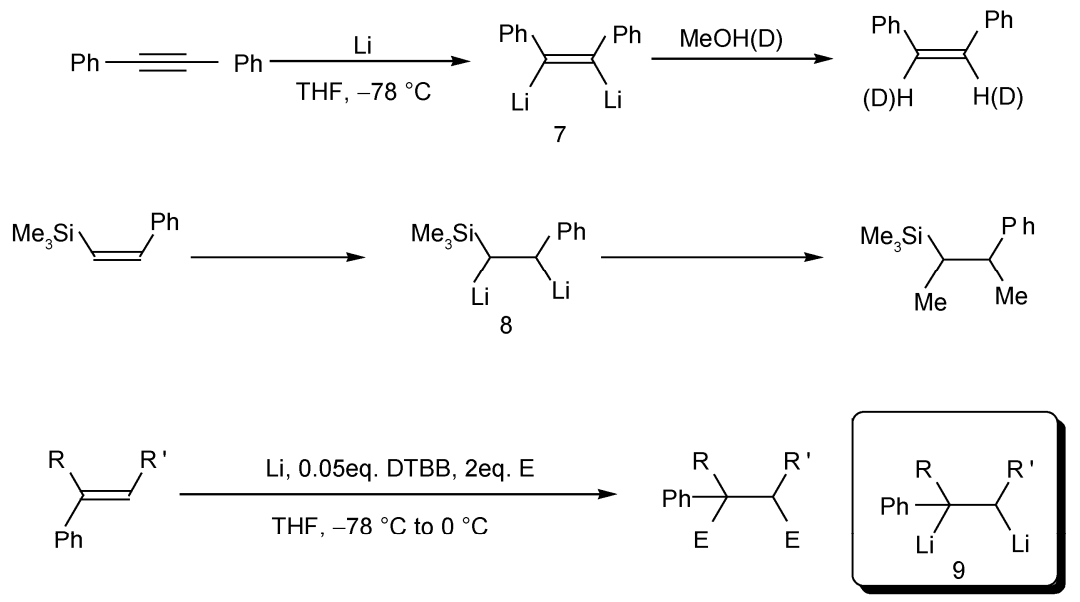

$\mathrm{R}=\mathrm{H}, \mathrm{Me} ; \mathrm{R}^{\prime}=\mathrm{H}, \mathrm{Me} ; \mathrm{E}=\mathrm{Me}_{3} \mathrm{SiCl}, \mathrm{Me}_{2} \mathrm{CO}, \mathrm{Et}_{2} \mathrm{CO}, n-\mathrm{Pr}_{2} \mathrm{CO},\left(\mathrm{CH}_{2}\right)_{5} \mathrm{CO}$

Scheme 4 Preparation and reactions of the 1,2-dilithio compounds.

dinuclear organolanthanide complexes. Upon reaction with $\mathrm{Me}_{2} \mathrm{GeCl}_{2}$ or $\mathrm{Me}_{2} \mathrm{SnBr}_{2}$ [15], 1,2-bis(chlorogermyl)carborane or 1,2-bis(bromostannyl)carborane $\mathbf{1 1}$ is generated, respectively. These two compounds can be converted into a several cyclic compounds (12 in Scheme 5) [16]. Mercuracarborands were prepared using dilithio carborane and they formed the porous material 13 with a new bonding motif by supramolecular self-assembly [17]. The dimerization of a 1,2-dicuprio carborane that was prepared in situ led to the formation of 1,1'-bis(o-carborane) 14 after hydrolysis [18]. Zirconium and hafnium substituted carboranes were also prepared and their structures confirmed by single crystal X-ray analysis [19].

A highly strained carborane-1,2-disilacyclobutene derivative $\mathbf{1 5}$ was prepared by a reaction between 1,2dichlorotetraalkyldisilane and dilithiated carborane. Exposure of this reactive compound to atmospheric oxygen led to the insertion of one oxygen atom between the $\mathrm{Si}-\mathrm{Si}$ bond [20]. Upon catalysis by $\mathrm{Pd}\left(\mathrm{PPh}_{3}\right)_{4}$, compound $\mathbf{1 5}$ reacted with substituted alkynes to afford the 6-membered cyclic products $\mathbf{1 6}$ (Scheme 5) [21].

In their research into the structure/reactivity relationships of lanthanacarboranes, Xie and coworkers [22] synthesized compounds 17 bearing a 6-, 7- and 8- membered inner ring. They investigated $\mathrm{C}-\mathrm{C}$ bond cleavage during the reductive processes. Hydroxyethyl- and alkoxyethyl-o- carboranes $\mathbf{1 8}$ were also prepared and were further treated with alkali metals to form special ligands for use with rare earth metal complexes (Scheme 5) [23].

Nickel-mediated regioselective $[2+2+2]$ cycloaddition reactions of carboryne with alkynes was found to provide the highly substituted benzocarboranes 19 and these are difficult to obtain by other methods [24]. Based on this discovery, nickel-mediated 3-component cycloaddition reactions of the carboryne with alkenes and alkynes through intermediate $\mathbf{2 0}$ afforded dihydrobenzocarborane derivatives with excellent chemo- and regioselectivity [25]. Treatment of $\mathrm{Cp}_{2} \mathrm{ZrCl}_{2}$ with 1 equivalent of $\mathrm{Li}_{2} \mathrm{C}_{2} \mathrm{~B}_{10} \mathrm{H}_{10}$ in $\mathrm{Et}_{2} \mathrm{O}$ at room temperature for $1 \mathrm{~h}$ gave a novel zirconocenecarboranyl complex (21 in Scheme 5) [26]. This complex was transformed to the corresponding zirconacyclopentene 22 by reaction with 3-hexyne in refluxing toluene (Scheme $6)$. It was found that 22 is a versatile intermediate for the synthesis of various carborane derivatives [27].

Lithiation catalyzed by DTBB can convert 2,3-dichlopropene into the corresponding dilithium derivative ( 23 in Scheme 7), and this was captured using two equivalents of symmetrically substituted ketones [28]. The methylenesubstituted diol product $\mathbf{2 4}$ was further converted into the 1,5-dioxaspiro[2.4]heptanes $\mathbf{2 5}$, which is a structural motif found in many biologically active natural products.

Vinyl dilithium compounds can be generated from the corresponding dihalogen compounds through halogen-lithium exchange [29]. Because the reactivity of the two halogen atoms are different, equivalents of the 1,2-dilithio compounds were developed by stepwise lithiation followed by a subsequent reaction. In the case of 2,3-dibromo- $N$-methylindole 26, two different electrophiles were introduced to the indole unit using this method (Scheme 8) [30].

Trans-1,2-bis(tributylstannyl)ethylene 27 was obtained using a two-step protocol as shown in Scheme 9. It can be converted to trans-1,2-bis-stannylcyclopropane $\mathbf{2 8}$ by palladium-catalyzed cyclopropanation (Scheme 9). Such a stereodefined cyclopropane dianion can be used as a building block in natural product synthesis [31].

\subsection{1,3-Bimetallic compounds}

Benzylacetylene can be converted to the corresponding 1,3-dilithio intermediate 29 by hydrogen-lithium exchange (Scheme 10). A reaction between the dilithiated alkyne and isothiocyanates followed by the successive addition of $t$-BuOH in DMSO and methyl iodide led to cyclobutene 30 


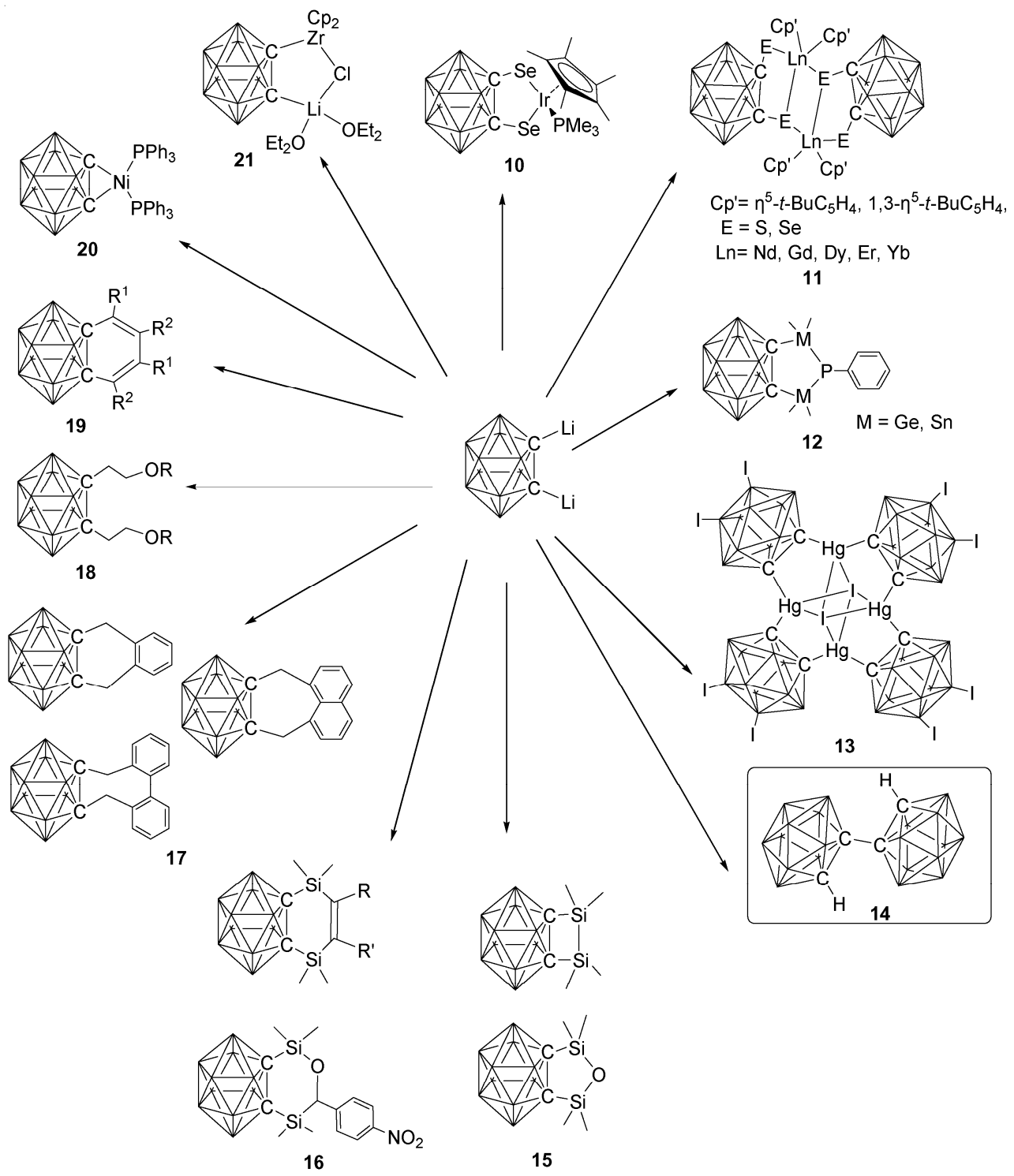

Scheme 5 Reactions of dilithio carborane.

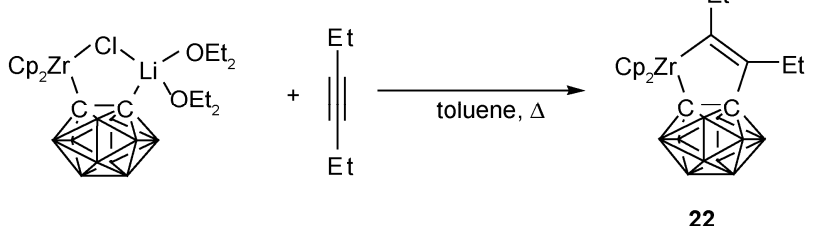

Scheme 6 Synthesis of intermediate 22

or thiacyclobutane $\mathbf{3 1}$ derivatives and this depended on the nature of the isothiocyanates [32]. Compound $\mathbf{3 1}$ was believed to be formed by a thermal rearrangement [33].

The lithiation of tribenzylidenemethane and diphenylacetone led to the formation of a Y-conjugated dianion (Scheme 11) and this was found to yield mono- and disubstituted products after quenching with a variety of electro- philes. A quench reaction between diphenylacetone dianion and TMSCl was found to form an allene [34].

Compound $\mathbf{3 3}$ cannot be obtained by direct halogen-lithium exchange. It was lithiated with excess lithium in the presence of a catalytic amount of naphthalene. As shown in Scheme 12, the dilithio species formed was captured by a carbonyl compound to afford the diol 34, which was converted to the perhydrofurofuran derivative $\mathbf{3 5}$ [35,36] or to the 1,6-dioxaspiro[3.4]octanes 36 [37].

Stable alkyl 1,3-dilithio compounds can be generated by the cleavage of phenyl substituted cyclopropanes in the presence of lithium metal or lithium di-t-butylbiphenyl, and they were further trapped by electrophiles. As shown in Chart 1, cleavage always occurred at the carbon-carbon bond next to the phenyl group [38].

The dilithiated indole derivative $\mathbf{3 8}$ was synthesized by the lithialation of $\mathbf{3 7}$ (Scheme 13), which was formed through 


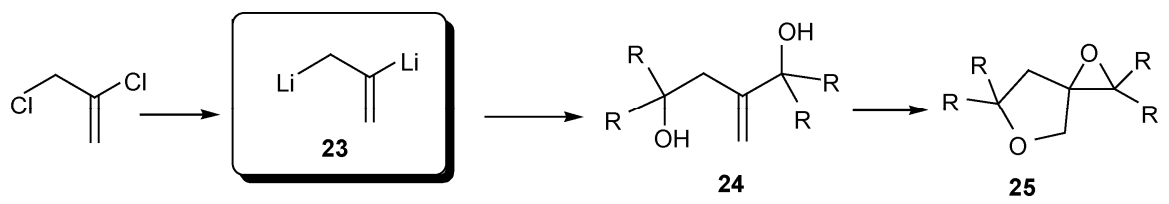

Scheme 7 Synthesis and reaction of 2,3-dilithiopropene.<smiles>Cn1c(Br)c(Br)c2ccccc21</smiles>

26<smiles>CCc1c(Cl)c2ccccc2n1CC</smiles>

Scheme 8 Synthesis of 2,3-dilithio- $N$-methylindole.

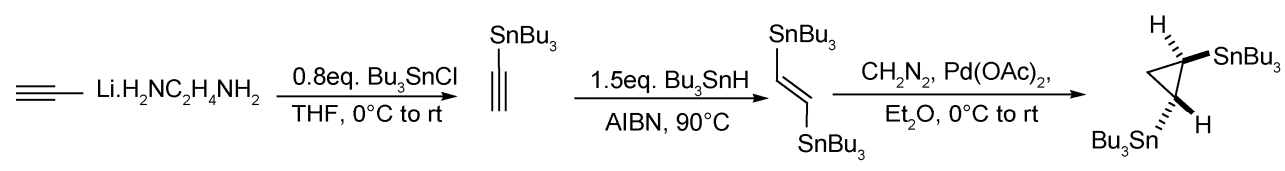

27
28

Scheme 9 Preparation of trans-1,2-bis-stannylcyclopropane.
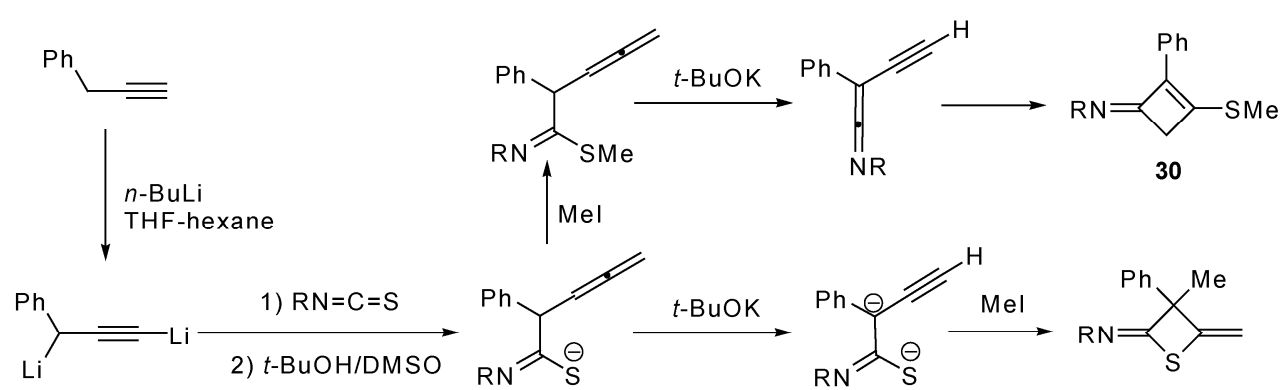

29

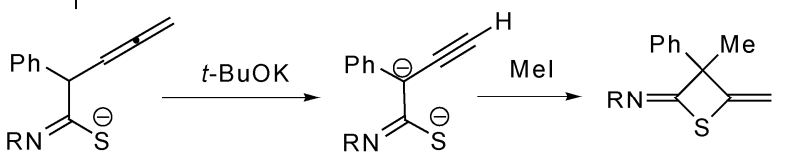

31

Scheme 10 Preparation and reactions of 1,3-dilithio benzylacetylene.

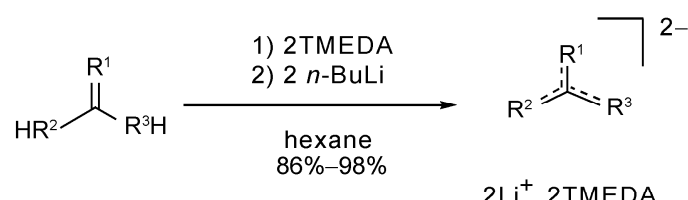

$2 \mathrm{Li}^{+}$2TMEDA<smiles>CCPCC(Cc1ccccc1)Cc1ccccc1</smiles>

$2 \mathrm{Li}^{+}$2TMEDA<smiles>[Z]CC(=CC(=O)C=Cc1ccccc1)c1ccccc1</smiles>

$2 \mathrm{Li}^{+}$2TMEDA

Scheme 11 Preparation of the Y-conjugated dianion.

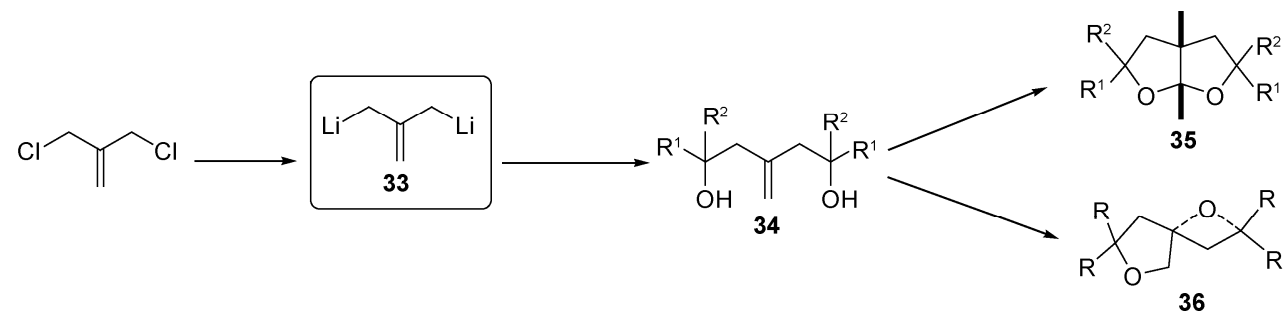

Scheme 12 Synthesis and application of the trimethylenemethane dianion. 
<smiles>c1ccc(C2(c3ccccc3)CC2)cc1</smiles>

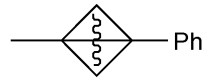

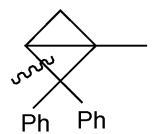<smiles>c1ccc(C2CC23CC3)cc1</smiles>

Chart 1 The cleaved $\mathrm{C}-\mathrm{C}$ bonds in the cyclopropanes.

intramolecular carbometalation and that reaction was either promoted by TMEDA or catalyzed by $\mathrm{CuCN}$. Because of the different reactivity of the two lithium atoms, two different electrophiles can be introduced into the indole skeleton in a stepwise manner [39,40].

\section{1,4 1,4-Bimetallic compounds}

(1) 1,4-Dilithio compounds. Among the 1,4-dilithio compounds, the 1,4-dilithio-1,3-butadiene derivatives that were mainly developed by Xi's group $[41,42]$ are a very special class of reagents because they often show unique reactivity with different electrophiles. Recently, Xi [43] thoroughly reviewed the reaction and synthetic applications of these reagents. A plausible explanation for this phenomenon is the cooperative effect between the two lithium atoms that are in close proximity. Single crystals of a trimeric 1,4-dilithio-1,3-butadiene and a dimeric $\mathrm{Me}_{3} \mathrm{Si}$-substituted 1,4-dilithio-1,3-butadiene were obtained with their structures confirmed by X-ray analyses [44].

It is widely accepted that ordinary organolithium compounds react with ketones or aldehydes to afford the corresponding alcohols. However, as shown in Scheme 14, when 1.4-dilithio-1,3-butadienes were used at low temperature, a variety of cyclopentadiene derivatives 39 or spiro compounds 40 were obtained [45]. It has been proposed that a chelation of the two alkenyllithium moieties with the carbonyl group was involved in the reaction followed by a stepwise attack on the carbonyl group and a concomitant loss of lithium oxide.

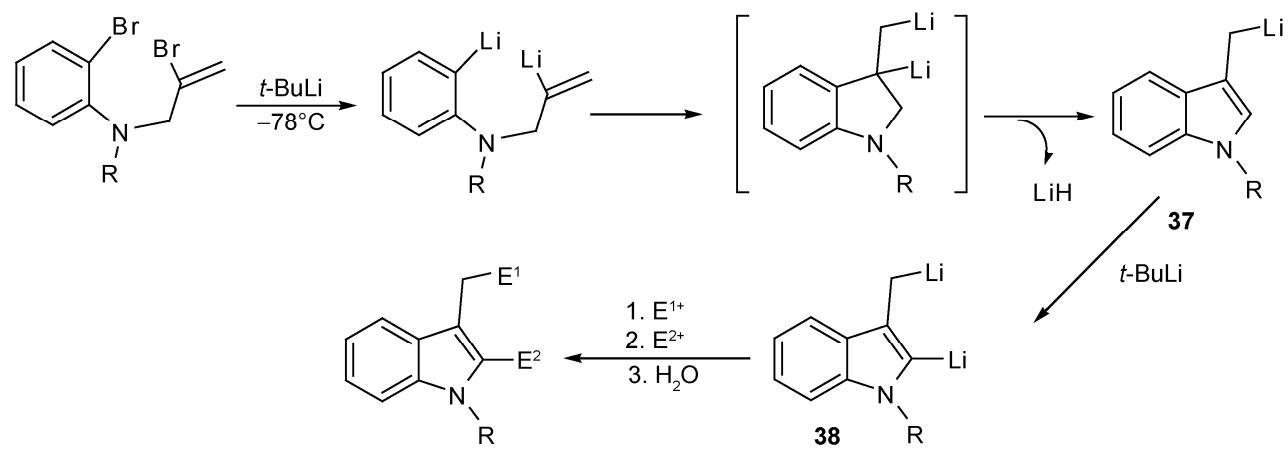

Scheme 13 Preparation of the dilithiated indole.<smiles>[R]C=C([R])[C@]1([R])O[C@H]([R7])C([R])=C1[R]</smiles>

41<smiles>[R]C1=C([R])C2(CCCCC2)C([R])=C1[R]</smiles>

40<smiles>[R]C1=C([R])C([R])C([R])=C1[R]</smiles>

39

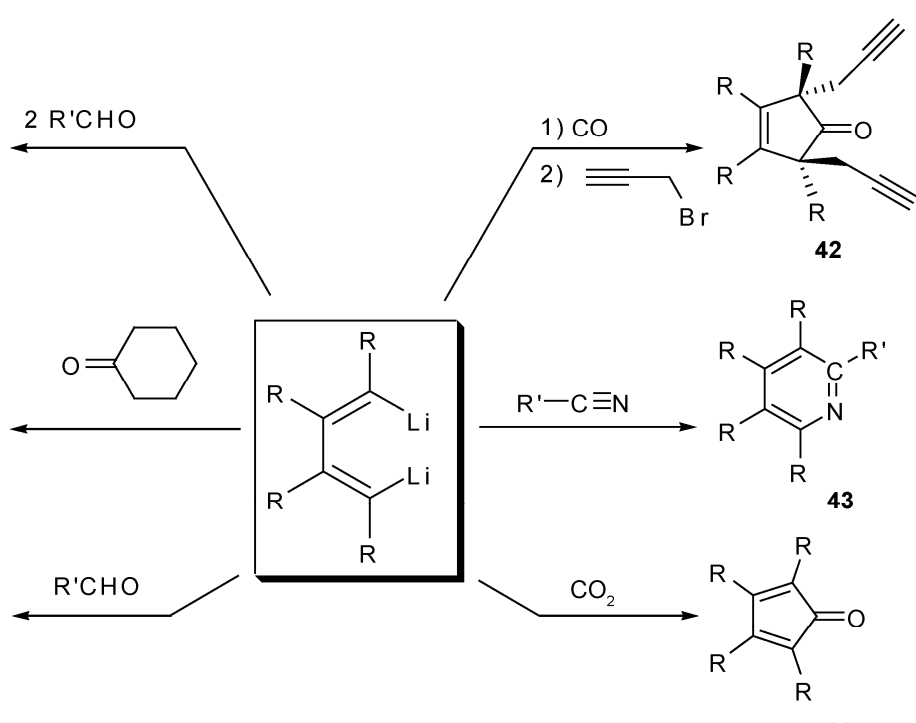

44

Scheme 14 Typical reactions of 1.4-dilithio-1,3-butadiene derivatives. 
Additionally, the reagent ratio affects the reaction patterns. For example, when 2 equivalents of aldehyde was treated with 1,4-dilithio-1,3-butadiene at room temperature, the highly regio- and stereoselective polysubstituted 2,5-dehydrofuran derivatives $\mathbf{4 1}$ were obtained (Scheme 14) [46].

3-Cyclopenten-1-ones $\mathbf{4 2}$ with a perfect trans configuration were successfully synthesized by the hydrolysis of a 1,4-dilithio-1,3-butadiene and $\mathrm{CO}$ reaction mixture at $-78^{\circ} \mathrm{C}$ for $1 \mathrm{~h}$ (Scheme 14). The corresponding derivatives were obtained in excellent yield after treating the reaction mixture with various electrophiles such as $\mathrm{MeI}$ and $\mathrm{Me}_{2} \mathrm{SO}_{4}$ [47]. Recently, the key intermediate of that reaction, an oxycyclopentadienyl lithium compound, was successfully isolated as a single crystal and its structure was fully characterized [48].

In the presence of HMPA, the reaction of nitriles with 1,4-dilithio-1,3-dienes gave the pyridines 43 (Scheme 14). Surprisingly, the same products can be obtained when 1-lithio-1,3-dienes are used [49]. These results are very different from the usual reaction between organolithium and nitriles as shown in standard textbooks where ketones or imines are formed. By changing the substituents on the butadienyl skeleton and applying different types of nitriles; pyridines, tricyclic $\Delta^{1}$-bipyrrolines, siloles or $(Z, Z)$-dienylsilanes were formed. The ratio of dilithio species vs. nitrile played an important role in reaction outcomes [50].

The hydrolysis of carbon dioxide and organolithium compound reaction mixtures gives acids although ketones are often detected as byproducts. However, the reaction between 1,4-dilithio-1,3-dienes with carbon dioxide was found to be a simple and effective way to prepare cyclopentadienones 44 (Scheme 14). ${ }^{13} \mathrm{C}$ NMR spectra showed that the final products were formed quantitatively before hydrolysis [51].
The reaction between 1,4-dilithio-1,3-dienes with $\mathrm{CS}_{2}$ is different from that with $\mathrm{CO}_{2}$ because the cleavage of the C-S double bonds afford multiple-substituted thiophenes and cycloaddition reactions that lead to thiopyran-2-thiones were observed [52]. This work provided a convenient method for the preparation of the S-containing heterocycles 45 although the exact reaction mechanism is still not clear (Scheme 15).

In the reaction between 1,4-dilithio-1,3-butadiene and dimethyl oxalate, varying the molar ratio of the reagents leads to the multiple substitution of $o$-benzoquinones or stereodefined 2,6-dioxabicyclo[3.3.0]-octa-3,7-dienes 46 as major products (Scheme 15) [53]. The order of reagent addition affects the distribution of the products.

As a special carbonyl compound, N,N-dimethylformamide (DMF) can be treated with a lithio compound or a Grignard reagent to form an aldehyde, which is known as the Bouveault reaction. This reaction works for certain 1,4-dilithio-1,3dienes and 2,5-dihydrofuran derivatives 47 were formed as byproducts (Scheme 15). For some other 1,4-dilithio-1,3-dienes, 2,5-dihydrofuran derivatives were the only isolated products. The substituents attached to the 1,4-dilithio-1,3-dienes determine the fate of the reactions [54,55].

By a cleavage of the $\mathrm{C}-\mathrm{S}$ bond, the iminocyclopentadiene derivatives $\mathbf{4 8}$ can be prepared in high yield by the reaction of 1,4-dilithiobutadienes with isothiocyanates (Scheme 15). Presumably, the cleavage takes place by a successive inter-intramolecular carbophilic addition [56]. The reaction of isocyanates with 1,4-dilithiobutadienes is very different from that of isothiocyanates. As a representative example of the later, a messy mixture containing at least 4 products was obtained. This difference was attributed to the high reactivity of the isocyanates.

Partially fluorinated naphthalene derivatives with multiple substituents (compound 49 in Scheme 15) were readily

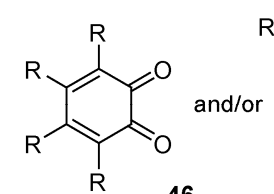

46
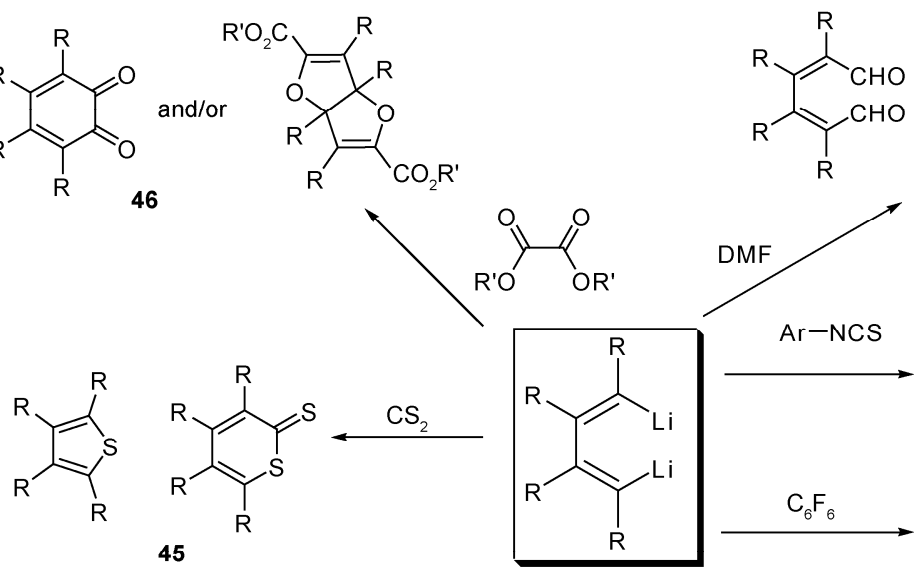

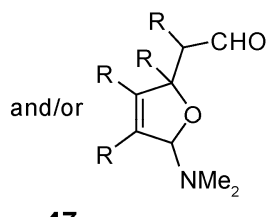

47<smiles>[R]C1=C([R])C([R])=C([R])C1=N[Al]</smiles>

48

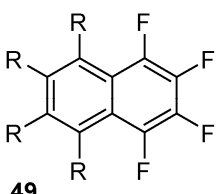

Scheme 15 Additional reactions of 1.4-dilithio-1,3-butadiene derivatives. 
prepared by the reaction of 1,4-dilithio-1,3-butadiene derivatives with hexafluorobenzene, and this was proposed to be the result of a sequential inter-intramolecular nucleophilic substitution [57]. For multi-substituted benzene bearing other halogen atoms such as chlorine or bromine a chlorine-lithium or bromine-lithium exchange reaction took place instead of a nucleophilic substitution even if only one fluorine atom in the hexafluorobenzene was substituted.

1,4-Dilithio-1,3-butadiene derivatives bearing trimethylsilyl groups at terminal carbons (compound $\mathbf{5 0}$ in Scheme 16) were found to be easily transformed into lithio siloles $\mathbf{5 1}$ by reflux in ether and in the presence of HMPA. These can undergo a series of reactions as general lithio reagents. Compared to the Tamao-Yamaguchi method for the preparation of lithio siloles, this route is more general in terms of substitution patterns and versatility [58-61]. Stable enols were successfully synthesized from similar 1,4-dilithio1,3-dienes and a novel de-aromatization/Michael addition/ re-aromatization domino process was believed to be involved in the reaction [62].

(2) 1,4-dicopper-1,3-diene. The reaction between alkynyl halides and 1,4-dicuprio-1,3-butadiene derivatives in a one-pot coupling protocol was reported in 1997. Highly substituted open chain 1,3-enynes $\mathbf{5 2}$ were obtained (Scheme 17). By a combination of successive coupling, unsymmetrically substituted dienynes were prepared in excellent yield [63].

Pentasubstituted arylcyclopentadienes and the fulvene derivatives $\mathbf{5 3}$ were prepared in moderate to good yields by the interaction of benzal halides or 1,1-dihalo alkene derivatives with 1,4-dicuprio-1,3-butadienes using similar procedures (Scheme 18) [64].

When the 1,4-dicuprio-1,3-dienes were treated at $50^{\circ} \mathrm{C}$, the octa-substituted semibullvalenes 54 (Scheme 18) were obtained in high yield [65]. This reaction provides an excellent synthetic method to obtain semibullvalenes, which is not easily accessible by other means. The semibullvalenes obtained can be converted quantitatively to the cyclooctatetraenes (COTs) 55 at 140 to $160^{\circ} \mathrm{C}$ [66].

Alkyl-substituted 1,4-dicuprio-1,3-dienes were found to undergo Michael addition followed by a ring-closure coupling reaction to give dihydro- 9,10 -anthraquinone derivatives, which were then oxidized by $p$-chloranil to the corresponding higher para-quinone 56 (Scheme 18). This work provided a novel one-pot method for the preparation of higher para-dihydroquinones and -quinones [67].

Unlike their dilithio analogues, 1,4-dicuprio-1,3-dienes gave the cyclopentadienones $\mathbf{5 7}$ and their head-to-head dimers 58 upon reaction with $\mathrm{CO}$. The two products resulted from intra- and intermolecular radical type reactions, respectively (Scheme 18) [68].

As shown in Scheme 19, the dimerization of alkyl-substituted 1,4-dicuprio-1,3-dienes at low temperature to form linear all-cis octatetraenes (compounds 59 and 60) and the tricyclo[4.2.0.0 $0^{2,5}$ octa-3,7-dienes derivatives (61 and 62) were reported in 2003 [69].

The fully-substituted COTs $\mathbf{6 3}$ were prepared by heating the twisted highly strained valence isomer of the cyclooctatetraene derivatives that were generated from 1,4-dicuprio-1,3-dienes (Scheme 20) [70]. Chen et al. [71] found that this transformation can also be achieved by adding benzoquinone to solutions of 1,4-dicuprio-1,3-dienes and cyclobutadiene is believed to be an important intermediated.

Chen et al. [72] also compared the effects of different oxidants in the oxidative coupling of 1,4-dicuprio-1,3-dienes including 1,4-benzoquinone, 1,4-naphthoquinone and p-chloranil. They found that naphthoquinone was a good oxidant for the generation of benzocyclobutadienes, which underwent intermolecular [4+2] cycloaddition to give dimers 64 (Scheme 21).

An investigation into the interaction between 1,4-dicopper-1,3-diene species and oxalyl chloride found that 1,1-cycloaddition products such as cyclopentadienones $\mathbf{6 5}$

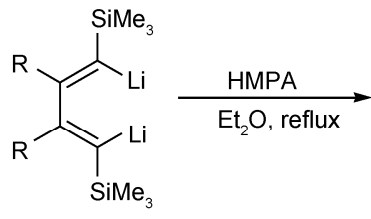

50<smiles>[R]c1c([SiH3])sc([AsH3])c1[R]</smiles>

51

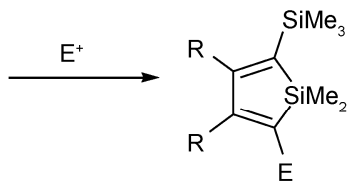

E

Scheme 16 Transformation of trimethylsilyl terminated 1,4-dilithio-1,3-butadienes.

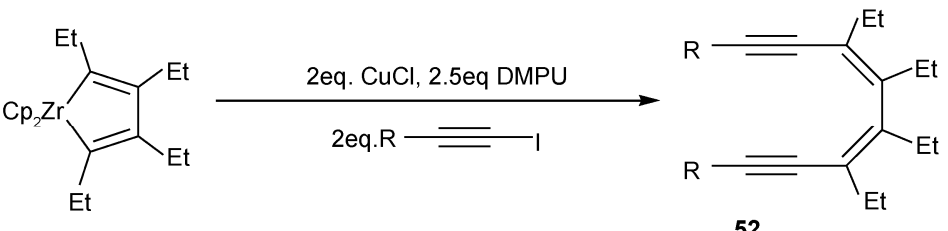

Scheme 17 Coupling reaction of 1,4-dicopper-1,3-butadiene. 


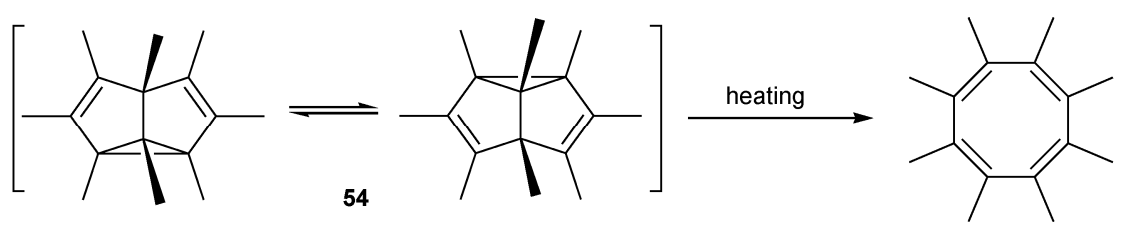

55

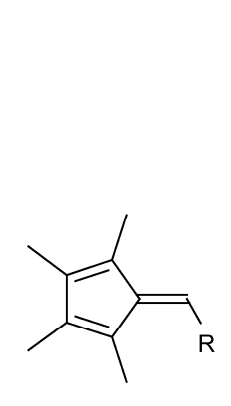

53
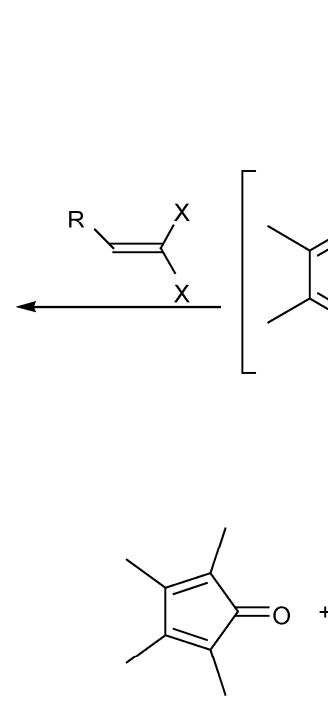

57

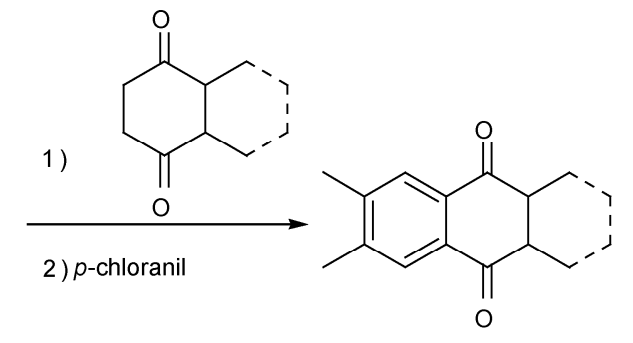

56
CO

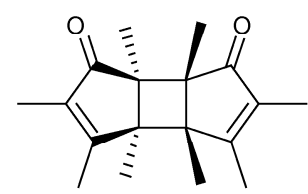

58

Scheme 18 Reactions of multi-substituted 1,4-dicuprio-1,3-dienes.

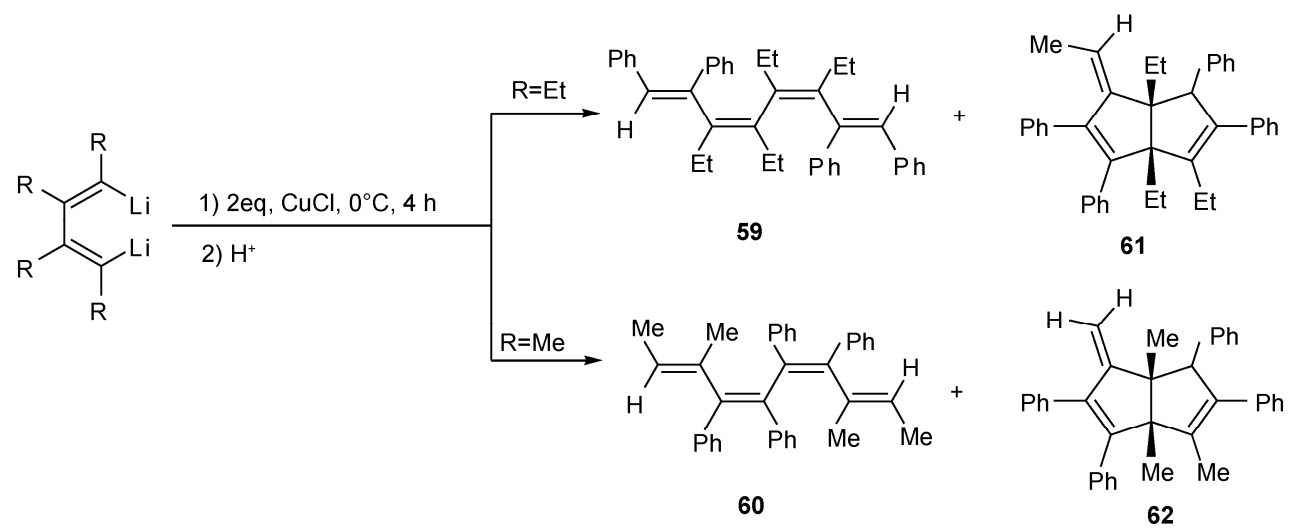

Scheme 19 The dimerization of alkyl-substituted 1,4-dicuprio-1,3-dienes.
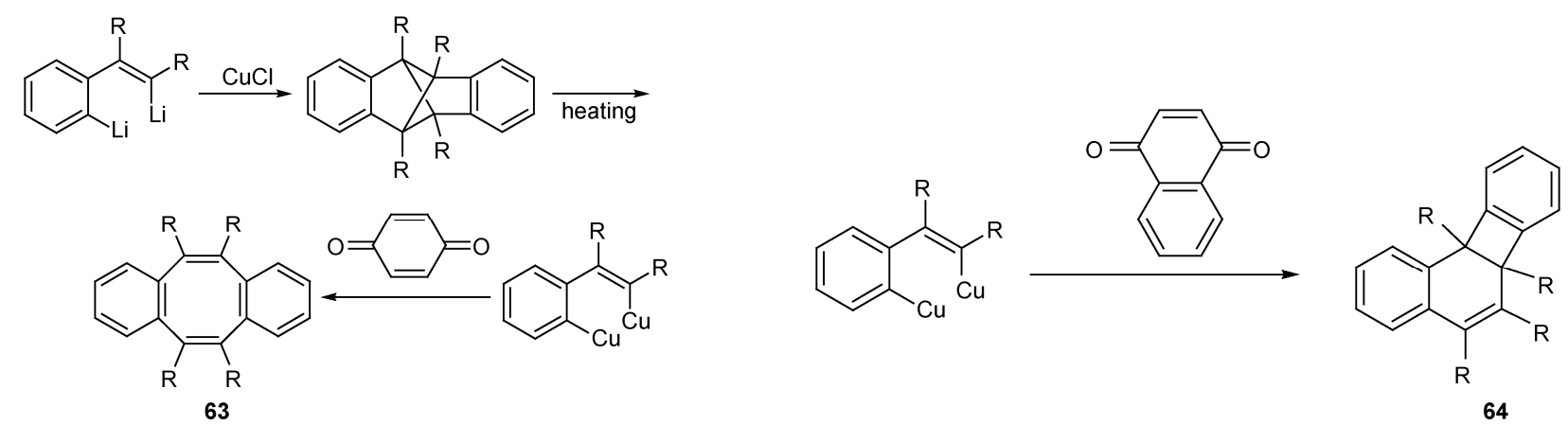

64

Scheme 20 Preparation of fully-substituted COT from 1,4-dicuprio-1,3-diene. Scheme 21 Intermolecular [4+2] cycloaddition of 1,4-dicuprio-1,3-dienes. 


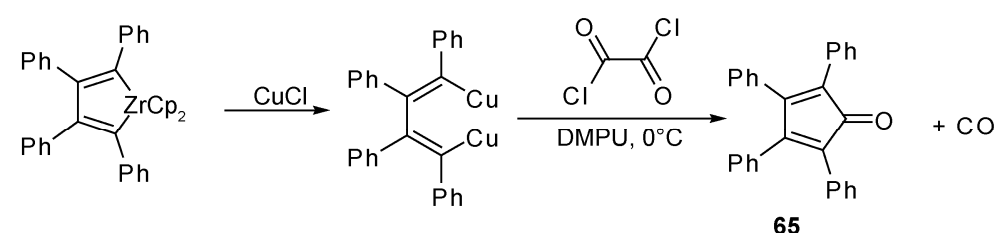

Scheme 22 Reaction between 1,4-dicopper-1,3-diene and oxalyl chloride.

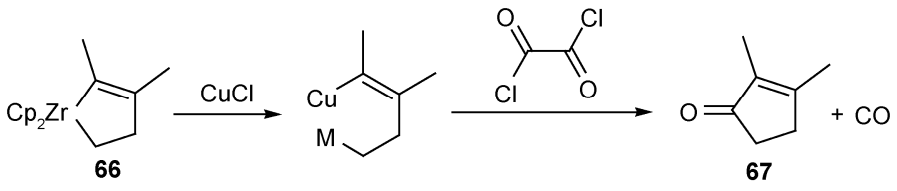

Scheme 23 Preparation of substituted cyclopentenones from zirconacyclopentenes.

were produced in moderate to high yields (Scheme 22) [73]. The chelating effect of the alkenylcopper moieties and the formation of $\mathrm{CO}$ gas were believed to be the driving force for the reaction. The mechanism was supported by the reaction of 1,4-dicopper-1,3-diene with methyl (chlorocarbonyl) formate under the same reaction conditions.

The reaction of zirconacyclopentenes $\mathbf{6 6}$ with oxalyl chloride in the presence of a catalytic amount of $\mathrm{CuCl}$ gives the cyclopentenones 67 (Scheme 23). This reaction represents a new and convenient way to produce substituted cyclopentenones using oxalyl chloride as the carbon monoxide source [74].

(3) Examples of other 1,4-dimetallic butadienes. Reactions between 1,4-bis(bromomagnesio)butadienes and carbonyl reagents were found to be different to those of 1,4-dilithio1,3-dienes. For example, under the same reaction conditions no reaction was observed between the 1,4-bis (bromomagnesio)butadienes and ketones. The magnesio-cyclopentadienes prepared from the 1,4-bis (bromomagnesio)butadienes were found to be more reactive. The $N$-Ph pyrroles ( 68 in Scheme 24) were obtained by reactions between PhNO and bromomanesio species [75].

The interaction between 1,4-dilithio-1,3-dienes with alkynes leads to the formation of benzene derivatives by mediation with a $2 / 3$ equivalent of $\mathrm{FeCl}_{3}$. No reaction was observed in the absence of $\mathrm{FeCl}_{3}$. Although the key intermediates were not isolated, the 1,4-diiron species 69 might be involved in the reaction (Scheme 25) [76].

The aluminacyclopentadienes $\mathbf{7 0}$, which can be considered equivalent to the dialumino species can be prepared

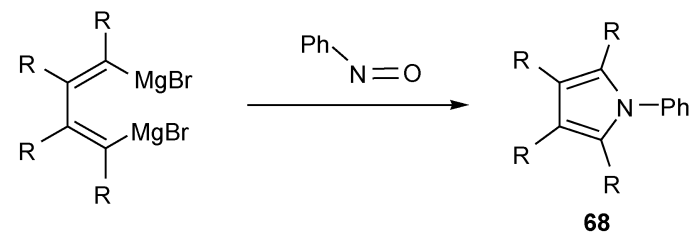

Scheme 24 Reaction between 1,4-bis(bromomagnesio)butadienes and PhNO. easily by a transmetalation of the corresponding 1,4-dilithio1,3-butadienes (Scheme 26). Their reactions with aldehydes at room temperature give cyclopentadiene derivatives including tetrahydroindenes in good to excellent yields [77].

\section{Homobimetallic catalysis for organic synthesis}

Enzymes are proteins with very high catalytic activities in biochemical reactions under mild conditions. To mimic enzymes in catalysis is highly desirable for chemists. Because some of the known enzymes have homobimetallic structures, dinuclear metal complexes such as dinuclear copper, dinuclear iron, dinuclear manganese and dinuclear nickel etc. [78] have been investigated in catalytic reactions [70]. According to their structures and the way they interact with reagents and substrates, dinuclear catalysts can be roughly classified into two types.

Van den Beuken and Feringa compiled a summary of enzymes with dinuclear active sites that cooperate with each other and they reviewed the design, synthesis and applications of dinuclear late transition metal complexes that showed promising catalytic activities [79]. They also modified the classification of dinuclear catalysts.

To some extent, the structure of the ligands determines the possibility of complex formation and their catalytic behavior. Maggini reviewed $\mathrm{P}, \mathrm{N}$-binucleating ligands in bimetallic complexes [80], Peris et al. [81] reviewed complexes with ( $N$-heterocyclic carbene) ligands while Gavrilova and Bosnich [78] established the principles of ligand design.

Although numerous bimetallic complexes have been synthesized, those that demonstrate catalytic activity are very rare. Many of these complexes have been designed and prepared for other purposes such as structural studies, luminescent property studies and host-guest chemistry etc. [82]. Here, we only discuss some typical examples of transition metal homobimetallic complexes in organic catalytic 


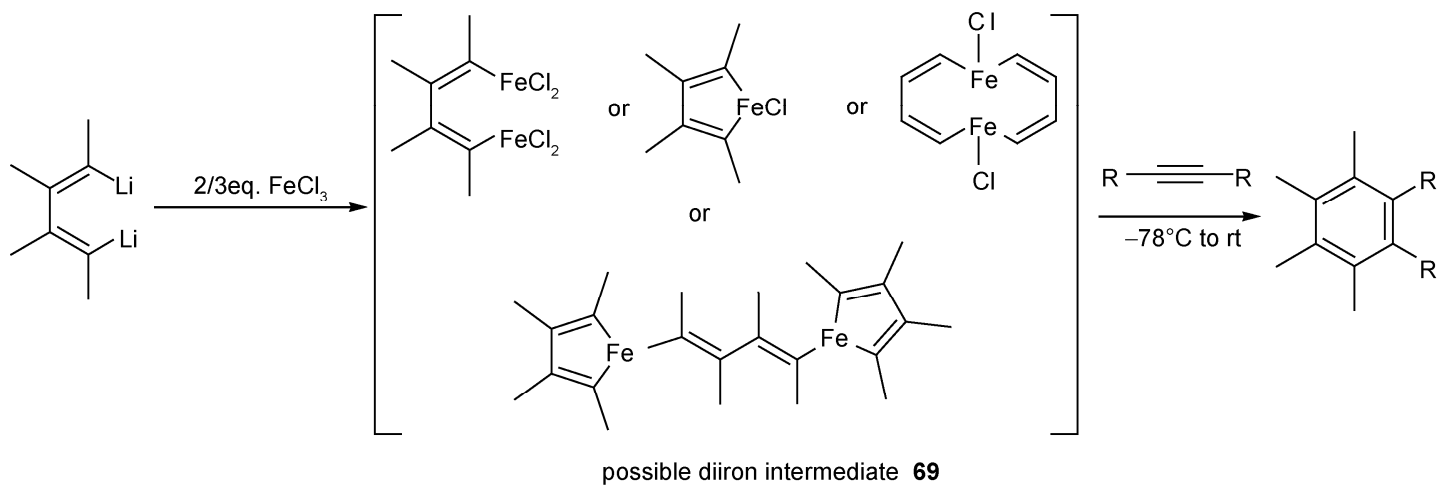

Scheme 25 Preparation of substituted benzene from 1,4-diiron-1,3-diene.

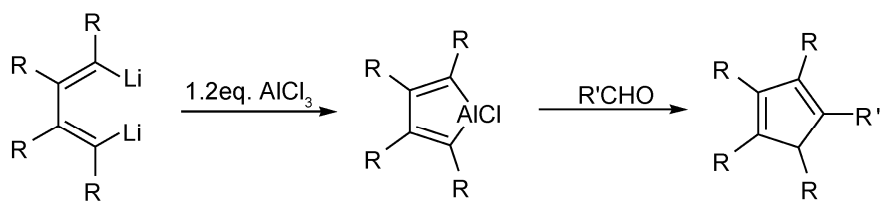

70

Scheme 26 Reaction between aluminacyclopentadienes and aldehydes.

reactions except for polymerization and oligomerization although many examples have also been included in the formerly mentioned excellent reviews.

\subsection{Dinuclear zinc catalyst}

Great progress has been made in catalytic asymmetric synthesis using the dinuclear zinc catalyst $\mathbf{7 1}$ developed by Trost and coworkers (Scheme 27). The catalyst was found to be effective in catalyzing the aldol reaction between aldehydes and aromatic ketones $\mathbf{7 2}$ [83] as well as $\alpha$-hydroxyketone 73 [84]. Its application was later extended to the catalysis of the aldol reaction between pyruvate-derived aldehyde and methyl ynones (equation 3 in Scheme 27), which provided tremendous flexibility for further structural manipulation [85]. Recently, the catalyst was found to be able to catalyze the asymmetric Michael addition to nitroalkenes to give synthetically versatile $\gamma$-substituted butenolides (eq. (4) in Scheme 27) [86]. Dinuclear zinc catalysts with similar structures were used for the catalyzed hydrolysis of $\beta$-lactam substrates, mimicking metallo- $\beta$ - lactamases in which two zinc ions are coordinated predominantly to histidine ligands [87].

As a nuclease mimic, calix[4]arene 76 (Chart 2) that contains two $\mathrm{Zn}$ (II) centers at the distal positions of the upper rim was synthesized and used in the hydrolysis of phosphate ester bonds. It showed high catalytic turnover number under very mild conditions. This catalyst was more effective than its monofunctionalized calix[4]arene counterpart 77, and this was attributed to the favorable contribution of the calix[4]arene moiety to substrate binding and the catalytic synergistic action of the two $\mathrm{Zn}$ (II) centers [88].

\subsection{Dinuclear ruthenium catalysts}

Dinuclear ruthenium catalysts were found to be active in catalyzing olefin metathesis reactions. Dias and Grubbs reported homobimetallic ruthenium catalysts $\mathbf{7 8}$ that were about 20 times more active than their monometallic counterparts although they were not as active as the heterobimetallic catalysts (Scheme 28) [89].

Recently, Sauvage et al. [90] reviewed the development of homobimetallic ruthenium catalysts and prepared novel homobimetallic ruthenium-(p-cymene) complexes bearing a tricyclohexylphosphine ligand and the polyunsaturated carbon-rich fragments 79 (Scheme 29). Complex 80 was found to be very active in catalyzing the ring closing metathesis (RCM) reaction of diallylmalonate and easily transformed to the Hoveyda-Grubbs catalyst $\mathbf{8 1}$ in high yield.

Viciano et al. [91] prepared the dinuclear Ru complex 82 bearing a triazolediylidene ligand and applied it to the $\alpha$-alkylation of secondary alcohols with primary alcohols (Scheme 30). Excellent conversions to the alcohols were obtained with a $1 \mathrm{~mol} \%$ catalyst loading in a short reaction time. By varying the procedure, the tetranuclear complex was obtained and this was also found to be active in the above-mentioned reaction.

\subsection{Examples of other bimetallic catalysts}

Shul'pin et al [92] summarized the activities of some Mncontaining systems during $\mathrm{H}_{2} \mathrm{O}_{2}$ dismutation and compared the performance of various Mn-containing catalysts in the oxidation of organic compounds with hydrogen peroxide. They found that a small amount of acetic acid was necessary for their dinuclear manganese complex 83 (Chart 3) to 

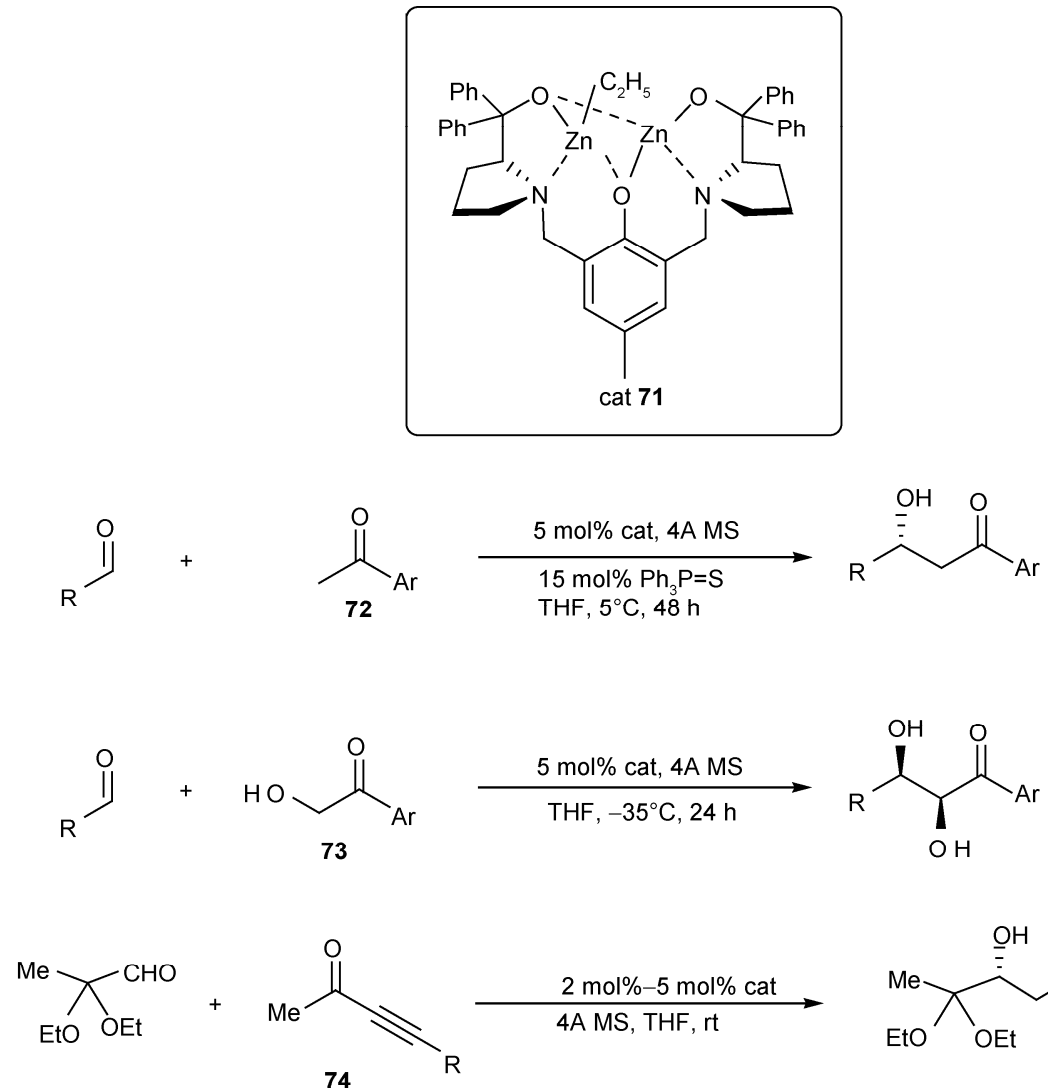<smiles>[R]C#CC(=O)C[C@@H](O)C(C)(OCC)OCC</smiles><smiles>[R]C=C[O+]=[N+]([O-])[O-]</smiles>

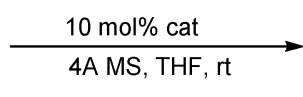<smiles>[R]C(C[N+](=O)[O-])[C@H]1C=CC(=O)O1</smiles>

Scheme 27 Reactions catalyzed by a dinuclear zinc catalyst.

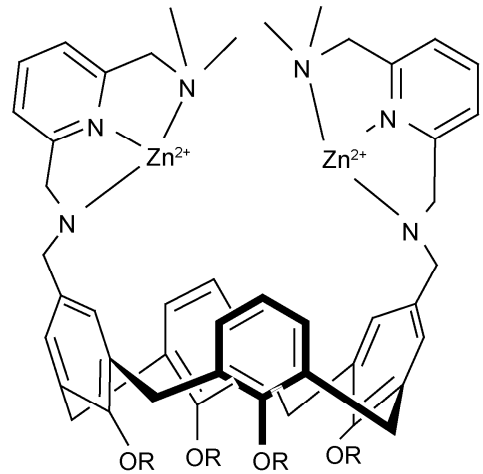

76

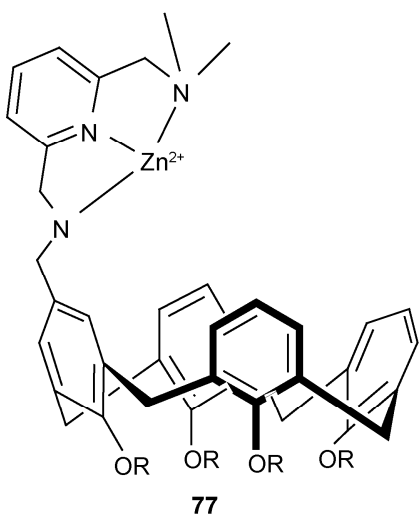

77

Chart 2 Structures of the zinc catalysts derived from calix[4]arene.

catalyze both hydrogen peroxide decomposition and cyclohexene oxygenation by $\mathrm{H}_{2} \mathrm{O}_{2}$. They extended the scope of these systems to the epoxidation of olefins, especially sterically accessible olefins including natural compounds like terpenes [93].
The dimeric (salen)titanium complex 84 (Chart 4) in which the titanium atoms are bridged by two oxygen atoms was formed in the asymmetric addition of trimethylsilyl cyanide to aldehydes and the dinuclear catalysts were very effective at ambient temperature. Water was found to be crucial 


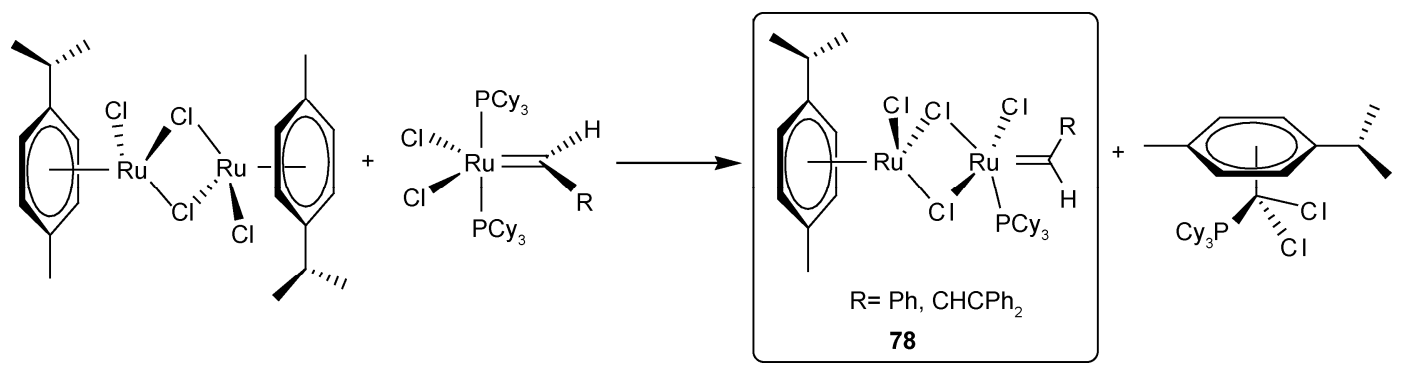

Scheme 28 Preparation of novel homobimetallic ruthenium catalysts.

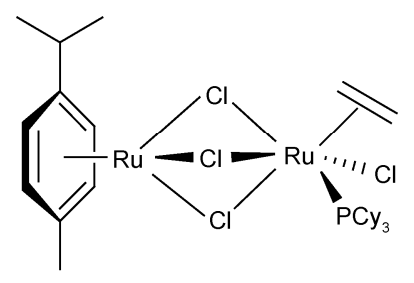<smiles></smiles>

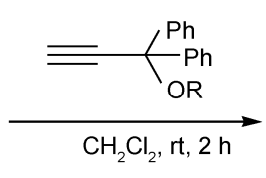

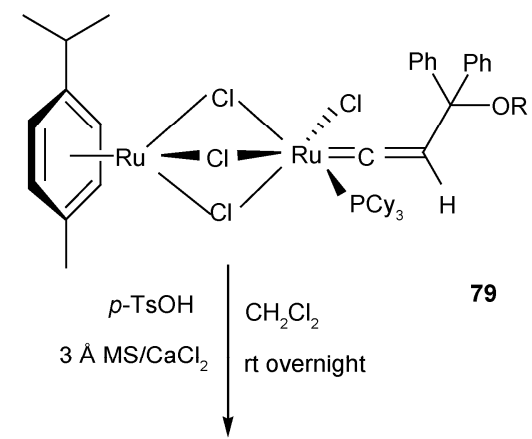<smiles>Cc1ccc(C(C)C)cc1</smiles><smiles>Cl[Te](Cl)(Cl)(Cl)(Cl)C1C=C(c2ccccc2)c2ccccc21</smiles>

Scheme 29 Transformation of the homobimetallic ruthenium catalysts.<smiles></smiles>

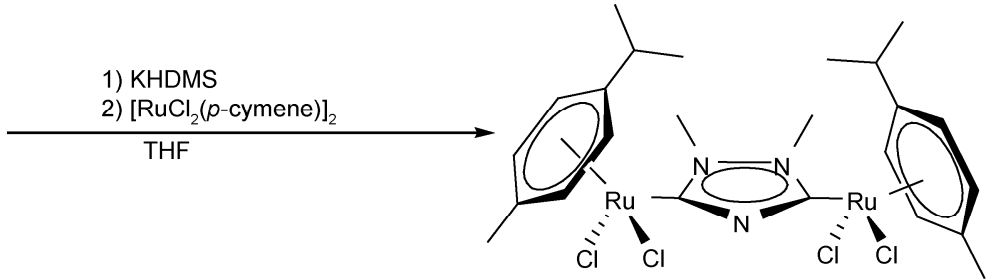

82

Scheme 30 Preparation of the dinuclear Ru complex bearing a triazolediylidene ligand.

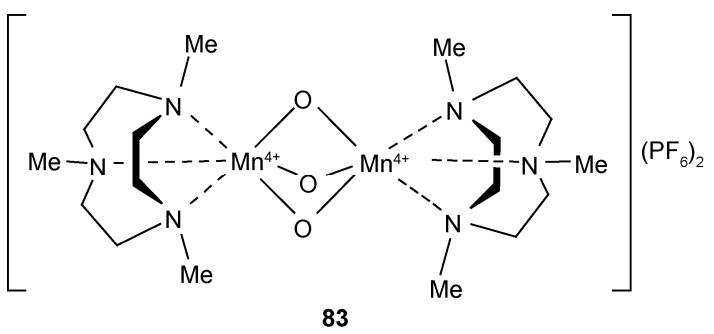

Chart 3 Structure of the dinuclear manganese catalyst used in $\mathrm{H}_{2} \mathrm{O}_{2}$ dismutation.

for the formation of the dimer [94]. Modified catalysts were prepared by linking the two parts with a spacer and the cooperative effect can be adjusted by changing the structure of the spacer. An exceptionally efficient chiral catalyst for the enantioselective cyanation of aldehydes was thus obtained [95].

A reaction between the $1,1^{\prime}$-dimethyl derivative of bitriazole with $\left[\mathrm{Rh}(\mathrm{CO})_{2}(\mathrm{OAc})\right]_{2}$ gave the dirhodium(II) complex 86 (Scheme 31). The structure was determined by $\mathrm{X}$-ray diffraction. This complex was found to be catalytically active in the transfer hydrogenation of acetophenone and benzophenone with $i-\mathrm{PrOH} / t-\mathrm{BuOK}$. The reactivity is similar to that of the monorhodium complex 87 [96].

The catalytic asymmetric preparation of chiral binaphthols was carried out by the oxidative coupling of 2-naphthols in the presence of the novel chiral oxovanadium(IV) complexes 88 (Chart 5). Target products with excellent 


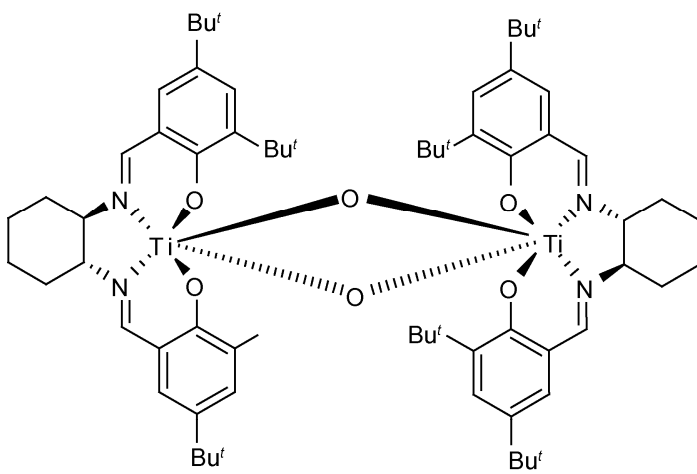

84

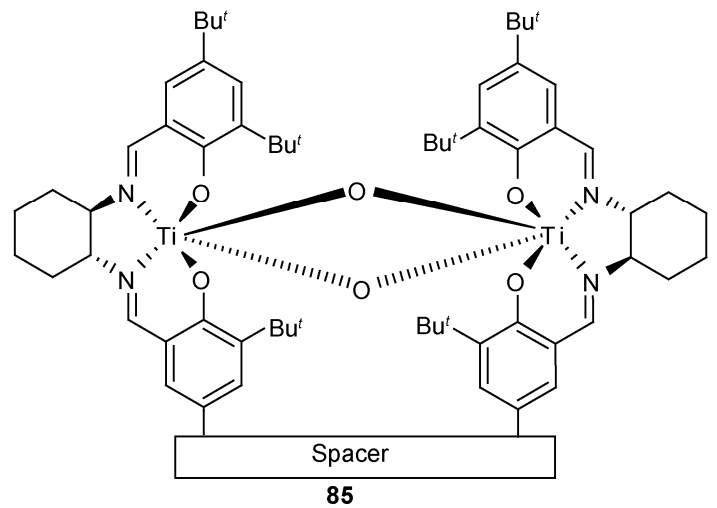

Chart 4 Structure of the dimeric (salen)titanium catalyst.

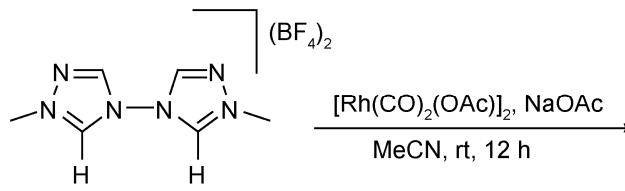

Scheme 31 Preparation of the dinuclear rhodium catalyst.

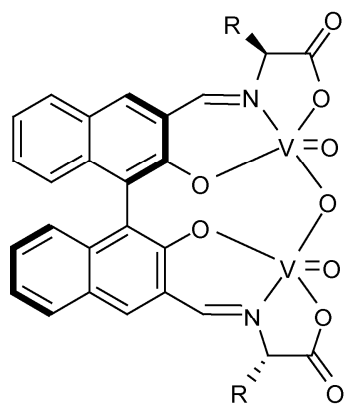

88

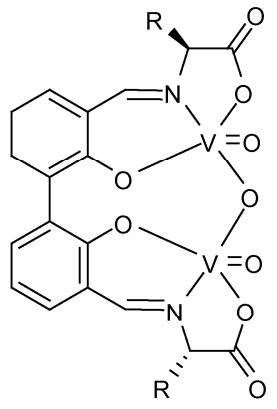

89
Chart 5 Structures of the chiral oxovanadium catalysts.

enantioselectivities were obtained in high yield [97]. Both the chiral centers on the amino acid part and the axially chiral binaphthyl unit were crucial for stereocontrol. Replacing the binaphthyl unit with a conformationally flexible biphenyl unit gave the diastereomeric oxovanadium(IV) complexes 89, which were also effective in the abovementioned reaction giving satisfactory yields and enantioselectivities [98].

The phenylene-bridged binuclear tetramethylcyclopentadienyl (Cp') organolanthanide complexes (90 and 91 in Chart 6) were synthesized and found to be effective in catalyzing the intramolecular hydroamination/cyclization of aminoalkenes, aminoalkynes and aminodienes. The distance between the lanthanide active centers was modulated by ortho-, meta- or para- substitution of the phenyl unit [99]. Detailed comparison studies on the stability and catalytic
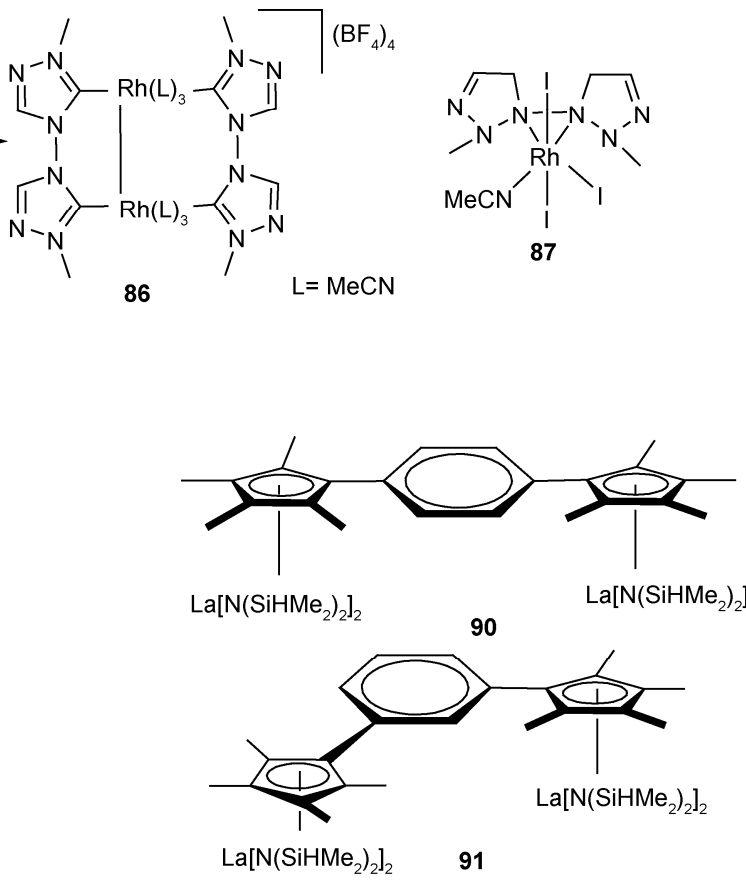

Chart 6 Structures of the phenylene-bridged organolanthanide complexes.

performance of the dinuclear complexes with those of mononuclear catalysts were also carried out [100].

Recently, Ritter and coworkers successfully obtained the Pd(III) complexes (92 and 93 in Chart 7) and confirmed their structures by X-ray crystallography. They showed that the $\mathrm{Pd}(\mathrm{III})$ complexes might be reaction intermediates in a class of Pd catalyzed reactions that had been believed to proceed via $\mathrm{Pd}(\mathrm{II}) / \mathrm{Pd}(\mathrm{IV})$ redox cycles [101-103].

\section{Summary}

From the reactions discussed in this review, the importance of homobimetallics in organic synthesis either as reagents or as catalysts is apparent. Plenty of evidence suggests that unique reaction patterns and the catalytic performance of 


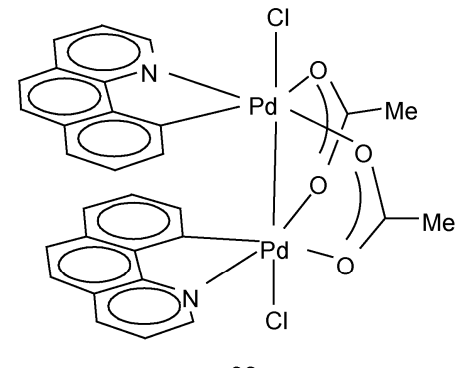

92

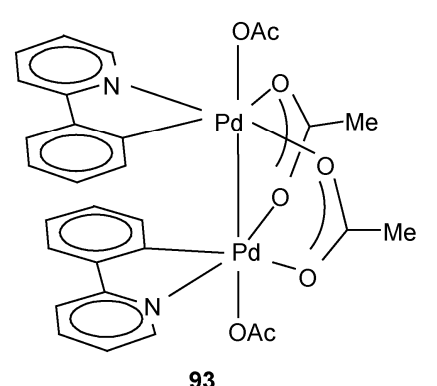

Chart 7 Structures of the synthesized Pd(III) complexes.

homobimetallics are caused by cooperative effects. Although the exact interactions are not clear to date, exciting discoveries in the application of homobimetallics could be expected. There is no doubt that studies on the mechanism of the cooperative effect will be both interesting and difficult.

This work was supported by the National Natural Science Foundation of China (20972025) and the China National Petroleum Corporation (CNPC) Innovation Foundation (2010D-5006-0504).

1 Thompson C M, Green D. Recent advances in dianion chemistry. Tetrahedron, 1991, 47: 4223-4285

2 Langer P, Freiberg W. Cyclization reactions of dianions in organic synthesis. Chem Rev, 2004, 104: 4125-4149

3 Foubelo F, Yus M. Organodilithium intermediates as useful dianionic synthons: Recent advances. Curr Org Chem, 2005, 9: 459-490

4 Marek I, Normant J F. Synthesis and reactivity of $\mathrm{sp}^{3}$-geminated organodimetallics. Chem Rev, 1996, 96: 3241-3267

5 Zarges W, Marsch M, Harms K, et al. $\left[\left\{\mathrm{Li}_{2}\left(\mathrm{Me}_{3} \mathrm{SiCCN}\right)\right\}_{12}\left(\mathrm{Et}_{2} \mathrm{O}\right)_{6}\right.$ $\left(\mathrm{C}_{6} \mathrm{H}_{14}\right)$ ] kristallstruktur mit dem trimethylsilylacetonitril-dianion. Chem Ber, 1989, 122: 1307-1311

6 Langer P, Döring M, Seyferth D, et al. Direct transformation of silyl enol ethers into functionalized allenes. Chem Eur J, 2001, 7: 573-584

7 Langer P, Döring M, Seyferth D, et al. Synthesis of nitrile oligomers through multiple anion capture reactions of allene dianions. Eur J Org Chem, 2003, 3: 1948-1953

8 Yus M, Gutiérrez A, Foubelo F. Polylithiation of thioethers: A versatile route for polyanionic synthons. Tetrahedron, 2001, 57: 4411-4422

9 Foubelo F, Gutiérrez A, Yus M. Methane-derived polyanionic synthons from bis(phenylthio)methane. Tetrahedron Lett, 1999, 40: $8177-8180$

10 Yoshino H, Toda N, Kobata M. Transition-metal-catalyzed sequential cross-coupling of bis(iodozincio)methane and ethane with two different organic halides. Chem Eur J, 2006, 12: 721-726

11 Maercker A, Kemmer M, Wang H C, et al. Mechanism of reduction of diphenylacetylene by metallic lithium. Angew Chem Int Ed, 1998, 37: $2136-2138$
12 Maercker A, Reider K, Girrese U. Polylithiumorganic compounds 24: The reaction of substituted vinylsilanes with lithium metal. Eur J Org Chem, 1998: 1455-1465

13 Yus M, Martínez P, Guijarro D. DTBB-catalysed dilithiation of styrene and its methyl-derivatives introduction of two electrophilic reagents. Tetrahedron, 2001, 57: 10119-10124

14 Herberhold M, Jin G X, Yan H, et al. The NMR-spectroscopic and $\mathrm{X}$-ray crystal-structural characterization of two $\mathrm{Cp} * \mathrm{Ir}$ half sandwich complexes containing the 1,2-Dicarba-closo-dodecaborane-1,2- diselenolato ligand. Eur J Inorg Chem, 1999, 873-875

$15 \mathrm{Yu} \mathrm{X} \mathrm{Y,} \mathrm{Jin} \mathrm{G} \mathrm{X,} \mathrm{Hu} \mathrm{N} \mathrm{H,} \mathrm{et} \mathrm{al.} \mathrm{Synthesis} \mathrm{and} \mathrm{structural} \mathrm{characteri-}$ zation of organolanthanide complexes with 1,2-dicarba-closo-dodecaborane-1,2-dichalcogenolate ligands. Organometallics, 2002, 21: $5540-5548$

16 Lee C, Lee J, Lee S W, et al. Synthesis and reactivity of 1,2-bis (chlorodimethylgermyl)carborane and 1,2-bis (bromodimethylstannyl) carborane. Inorg Chem, 2002, 41: 3084-3090

17 Lee H, Knobler C B, Hawthorne M F. Modular self-assembly of a microporous solid based upon mercuracarborand- 4 and a new bonding motif. Angew Chem Int Ed, 2001, 40: 2124-2126

18 Ren S, Xie Z. A Facile and practical synthetic route to $1,1^{\prime}$-Bis (o-carborane). Organometallics, 2008, 27: 5167-5168

19 Sit M M, Chan H S, Xie Z. Metallacarboranes incorporating an arachno- $\eta^{6}-C_{2} B_{9}$ ligand. Organometallics, 2009, 28: 5998-6002

20 de Rege F M, Kassebaum J D, Scott B L, et al. Synthesis structure and reactivity of $1,2-\left(1^{\prime}, 1^{\prime}, 2^{\prime}, 2^{\prime}\right.$-tetramethyldisilane-1', $\left.2^{\prime}\right)$ carborane. Inorg Chem, 1999, 38: 486-489

21 Song K H, Jung I, Lee S S, et al. Palladium-catalyzed double-silylation reactions of 3,4-Carboranylene-1,1,2,2-tetraethyl-1,2disilacyclobut-3-ene. Organometallics, 2001, 20: 5537-5541

$22 \mathrm{Zi} \mathrm{G}, \mathrm{Li} \mathrm{H} \mathrm{W}$, Xie Z. Synthesis, structural characterization and reactivity of 'carbons-adjacent' nido- and arachno-carborane anions of the $\mathrm{C}_{2} \mathrm{~B}_{10}$ systems and their metal complexes. Organometallics, 2002, 21: $5415-5427$

23 Cheung M S, Chan H S, Xie Z. Synthesis and structural characterization of hydroxyethyl and alkoxyethyl-o-carboranes and their alkali and rare earth metal complexes. Organometallics, 2004, 23: 517-526

24 Deng L, Chan H S, Xie Z. Nickel-mediated regioselective [2+2+2] cycloaddition of carboryne with alkynes. J Am Chem Soc, 2006, 128: 7728-7729

25 Qiu Z, Xie Z. Nickel-mediated three-component cycloaddition reaction of carboryne alkenes and alkynes. J Am Chem Soc, 2009, 131: 2084-2085

26 Deng L, Chan H S, Xie Z. Synthesis structure and reactivity of a zirconocene-carboryne precursor. J Am Chem Soc, 2005, 127: 1377413775

27 Ren S, Chan H S, Xie Z. Synthesis structure and reactivity of zirconacyclopentene incorporating a carboranyl unit. J Am Chem Soc, 2009, 131: 3862-3863

28 Alonso F, Meléndez J, Yus M. Synthesis of substituted 1,5-dioxaspiro [2.4]heptanes from 2,3-dichloroprop-1-ene. Helv Chim Acta, 2002, 85: 3262-3271

29 Liu Y, Gribble G W. Generation and reactions of 2,3-dilithio- $N$-methylindole. Synthesis of 2,3-disubstituted indoles. Tetrahedron Lett, 2001, 42: 2949-2951

30 Liu Y, Gribble G W. Selective lithiation of 2,3-dibromo-1-methylindole. A synthesis of 2,3-disubstituted indoles. Tetrahedron Lett, 2002, 43: 7135-7137

31 Heureux N, Marchant M, Maulide N, et al. Preparation and applications of a novel bis(tributylstannyl)cyclopropane: A synthetic equivalent of a cyclopropane-1,2-dianion. Tetrahedron Lett, 2005, 46: 79-83

32 Brandsma L, Spek A L, Trofimov B A, et al. Enxpected formation of derivatives of cyclobutene and thiacyclobutane from the reaction between dilithiated benzylacetylene and isothiocyanates. Tetrahedron Lett, 2001, 42: 4687-4689

33 Tarasova O A, Nedolya N A, Brandsma L, et al. A novel thermal rearrangement of allenic imidothioates Formation of iminocyclobute- 
nes. Tetrahedron Lett, 2004, 45: 5881-5883

34 Witt O, Mauser H, Friedl T, et al. Reactions of the lithium salts of the tribenzylidenemethane dianion, diphenylacetone dianion, and related compounds. J Org Chem, 1998, 63: 959-967

35 Alonso F, Lorenzo E, Yus M. Substituted 1,7-dioxabicycio [3.3.0] octanes: New easy access to the perhydrofurofuran core of aflatoxins and analogues. Tetrahedron Lett, 1997, 38: 2187-2190

36 Lorenzo E, Alonso F, Yus M. New trimethylenemethane dianion synthons: application to the preparation of substituted perhydrofuro[2,3-b]furans. Terahedron, 2000, 56: 1745-1757

37 Alonso F, Falvello L R, Fanwick P E, et al. Synthesis of substituted 1,6-dioxaspiro[3.4] octanes from 3-chloro-2-(chloromethyl)prop-1ene. Synthesis, 2000, 949-952

38 Maercker A, Oeffner K S, Girreser U. Polylithiumorganic compounds. Part 29: C-C Bond cleavage of phenyl substituted and strained carbocycles using lithium metal. Tetrahedron, 2004, 60: 8245-8256

39 Barluenga J, Sanz R, Granados A, et al. First intramolecular carbometalation of lithiated double bonds, a new synthesis of functionalized indoles and dihydropyrroles. J Am Chem Soc, 1998, 120: 4865-4866

40 Fañanás F J, Granados A, Sanz R, et al. Synthesis of functionalized pyrrole and indole derivatives through carbometallation of lithiated double bonds. Chem Eur J, 2001, 7: 2896-2907

$41 \mathrm{Xi} Z$ Z. Reaction chemistry of 1,4-dilithio-1,3-diene and 1-lithio-1,3diene derivatives. Eur J Org Chem, 2004, 2773-2781

42 Zhang W X, Xi Z. Synthetic methods for multiply substituted butadiene-containing building blocks. Synlett, 2008, (17): 2557-2570

43 Xi Z. 1,4-Dilithio-1,3-dienes: Reaction and synthetic applications. Acc Chem Res, 2010, 43: 1342-1351

44 Liu L, Zhang W X, Luo Q, et al. Isolation and X-ray structure of a trimeric 1,4-dilithio-1, 3-butadiene and a dimeric $\mathrm{Me}_{3} \mathrm{Si}$-substituted 1,4-dilithio-1,3-butadiene. Organometallics, 2010, 29: 278-281.

45 Xi Z, Song Q, Chen J, et al. Dialkenylation of carbonyl groups by alkenyllithium compounds: Formation of cyclopentadiene derivatives by the reaction of 1,4-dilithio-1,3-dienes with ketones and aldehydes. Angew Chem Int Ed, 2001, 40: 1913-1916

46 Chen J, Song Q, Li P, et al. Stereoselective synthesis of polysubstituted 2,5-dihydrofurans from reaction of 1,4-dilithio-1,3-dienes with aldehydes. Org Lett, 2002, 4: 2269-2271

47 Song Q, Chen J, Jin X, et al. Highly regio- and stereoselective 1,1-cycloaddition of carbon monoxide with 1,4-dilithio-1,3-dienes. Novel synthetic methods for 3-cyclopenten-1-one derivatives. J Am Chem Soc, 2001, 123: 10419-10420

48 Liu L, Zhang W X, Wang C, et al. Isolation, structural characterization and synthetic application of oxy-cyclopentadienyl dianions. Angew Chem Int Ed, 2009, 48: 8111-8114

49 Chen J, Song Q, Wang C, et al. Novel cycloaddition of nitriles with monolithio- and dilithiobutadienes. J Am Chem Soc, 2002, 124: 6238-6239

50 Yu N, Wang C, Zhao F, et al. Diversified reaction chemistry of 1,4-dilithio-1,3-dienes with nitriles: Fine-tuning of reaction patterns by substituents on the butadienyl skeleton leading to multi-substituted pyridines and/or $\Delta^{1}$-pyrrolines. Chem Eur J, 2008, 14: 5670-5679

51 Xi Z, Song Q. Efficient synthesis of cyclopentadieneone derivatives by the reaction of carbon dioxide with 1,4-dilithio-1,3-dienes. J Org Chem, 2000, 65: 9157-9159

52 Chen J, Song Q, Xi Z. Novel reaction patterns of carbon disulfide with organolithium compounds via cleavage of $\mathrm{C}=\mathrm{S}$ bonds or via cycloaddition reactions. Tetrahedron Lett, 2002, 43: 3533-3535

53 Mao G, Lu J, Xi Z. Formation of 2,6-dioxabicyclo[3.3.0]-octa-3,7dienes or multiply substituted $o$-benzoquinones from reactions of 1,4-dilithio-1,3-dienes with dimethyl oxalate. Tetrahedron Lett, 2004, 45: 8095-8098

54 Wang C, Mao G, Wang Z, et al. Facile synthesis of multiply substituted cyclopentadienes and conjugated dienals from the reaction of 1,4-dilithio-1,3-dienes with carboxylic acid derivatives including acid chlorides, anhydrides and DMF. Eur J Org Chem, 2007: 1267- 1273
55 Mao G, Wang C, Chen J, et al. Concise synthesis of multiply substituted stereodefined cis,cis-2,4-diene-1,6-dials,cis,cis-2,4-diene-1, 6diols and further applications. Tetrahedron Lett, 2005, 46: 53695372

56 Wang C, Song Q, Xi Z. Reactions of 1,4-dilithiobutadienes with isothiocyanates: Preparation of iminocyclopentadiene derivatives via cleavage of the $\mathrm{C}=\mathrm{S}$ double bond of a $\mathrm{RN}=\mathrm{C}=\mathrm{S}$ molecule. Tetrahedron, 2004, 60: 5207-5214

57 Wang Z, Wang C, Xi Z. Partially fluorinated naphthalene derivatives from 1,4-dilithio-1,3-dienes and $\mathrm{C}_{6} \mathrm{~F}_{6}$. Tetrahedron Lett, 2006, 47: 4157-4160

58 Wang C, Luo Q, Sun H, et al. Lithio siloles: Facile synthesis and applications. J Am Chem Soc, 2007, 129: 3094-3095

59 Luo Q, Gu L, Wang C, et al. Synthesis of functionalized siloles from Si-tethered diynes. Tetrahedron Lett, 2009, 50: 3213-3215.

60 Luo Q, Wang C, Gu L, et al. Formation of $\alpha$-lithio siloles from silylated 1,4-dilithio-1,3-butadienes: Mechanism and applications. ChemAsian J, 2010, 5: 1120-1128

61 Wang Z, Fang H, Xi Z. Cleavage of C-Si bond by intramolecular nucleophilic attack: Lithiation-promoted formation of siloles from 1-bromo-4-tetrasubstituted silyl-1,3-butadiene derivatives. Tetrahedron Lett, 2005, 46: 499-501

$62 \mathrm{Hu} \mathrm{Q}$, Wang $\mathrm{C}$, Li $\mathrm{D}$, et al. Formation of stable enols from 1,4-dilithio-1,3-dienes and acid chlorides by a de-aromatization/ Michael addition/re-aromatization domino process. Org Biomol Chem, 2007, 5: 2114-2118

63 Hara R, Liu Y, Sun W H, et al. Highly substituted enyne formation by coupling reaction of alkenylzirconium compounds with alkynyl halides. Tetrahedron Lett, 1997, 38: 4103-4106

64 Duan Z, Sun W H, Liu Y, et al. Formation of cyclopentadiene derivatives by reaction of zirconacyclopentadienes with 1,1-dihalo compounds. Tetrahedron Lett 2000, 41: 7471-7474

65 Wang C, Yuan J, Li G, et al. Metal-mediated efficient synthesis, structural characterization and skeletal rearrangement of octa-substituted semibullvalenes. J Am Chem Soc, 2006, 128: 4564-4565

66 Wang C, Xi Z. Metal mediated synthesis of substituted cyclooctatetraenes. Chem Commun, 2007, 5119-5133

67 Chen $\mathrm{C}, \mathrm{Xi} \mathrm{C}$, Ai Z, et al. Cycloaddition reaction of zirconacyclopentadienes to quinones: Synthesis of higher para-quinones. Org Lett, 2006, 8: 4055-4058

68 Luo Q, Wang C, Zhang W X, et al. CuCl-mediated tandem CO insertion and annulation of 1,4-dilithio-1,3-dienes: Formation of multiply substituted cyclopentadienones and/or their head-to-head dimmers. Chem Commun, 2008, 1593-1595

69 Li G, Fang H, Xi Z. Formation of stereodefined multiply substituted all-cis octatetraenes tricycle-[4.2.0.0 $0^{2,5}$ octa-3,7-dienes and pentalenes via $\mathrm{CuCl}$ or $\mathrm{FeCl}_{3}$-mediated dimerization of 1-lithiobutadienes and 1,4-dilithiobutadienes. Tetrahedron Lett, 2003, 44: 8705-8708

70 Li G, Fan H, Zhang S, et al. Synthesis, structural characterization, and skeletal rearrangement of dibenzo tricyclo[3.3.0.02,6]-1,2,5,6tetrasubstituted-octanes. Tetrahedron Lett, 2004, 45: 8399-8402

71 Chen C, Xi C, Lai C, et al. Coupling reactions of 1,4-dicuprio-1,3dienes: Formation of carbocycles. Eur J Org Chem, 2004: 647-650

72 Chen C, Xi C, Liu Y, et al. Generation of benzocyclobutadiene derivatives from zirconaindene derivatives. J Org Chem, 2006, 71: 5373-5376

73 Chen C, Xi C, Jiang Y, et al. 1,1-Cycloaddition of oxalyl dichloride with dialkenylmetal compounds: Formation of cyclopentadienone derivatives by the reaction of 1,4,-dilithio-1,3-dienes or zirconacyclopentadienes with oxalyl chloride in the presence of $\mathrm{CuCl}$. J Am Chem Soc, 2005, 127: 8024-8025

74 Chen C, Liu Y, Xi C. CuCl-catalyzed reaction of zirconacyclopentenes with oxalyl chloride: A new pathway for the preparation of cyclopentenones. Tetrahedron Lett, 2009, 50: 5434-5436

75 Fang H, Li G, Mao G, et al. Reactions of substituted (1,3-butadiene1,4-diyl) magnesium, 1,4-bis(bromomagnesio)butadienes and 1,4-dilithiobutadienes with ketones, aldehydes and PhNO to yield cyclopentadiene derivatives and $\mathrm{N}-\mathrm{Ph}$ pyrroles by cyclodialkenyla- 
tion. Chem Eur J, 2004, 10: 3444-3450

$76 \mathrm{Li} \mathrm{G}$, Fang $\mathrm{H}$, Li Z, et al. $\mathrm{FeCl}_{3}$-mediated reaction of 1,4-dilithio-1,3dienes with alkynes affording benzene derivatives. Chin J Chem, 2003, 219-221

77 Fang H, Zhao C, Li G, et al. Reaction of aluminacyclopentadienes with aldehydes affording cyclopentadiene derivatives. Tetrahedron, 2003, 59: 3779-3786

78 Gavrilova A L, Bosnich B. Principles of mononucleating and binucleating ligand design. Chem Rev, 2004, 104: 349-383

79 Steinhagen H, Helmchen G. Asymmetric two-center catalysis learning from nature. Angew Chem Int Ed Eng, 1996, 35: 2339-2342

80 Van den Beuken E K, Feringa B L. Bimetallic catalyssis by late transition metal complexes. Tetrahedron, 1998, 54: 12985-13011

81 Poyatos M, Mata J A, Peris E. Complexes with poly $(N$-heterocyclic carbene) ligands: Structural features and catalytic applications. Chem Rev, 2009, 109: 3677-3707

82 Maggini S. Classification of P,N-binucleating ligands for hetero and homobimetallic complexes. Coordin Chem Rev, 2009, 253: 1793 1832

83 Trost B M, Ito $\mathrm{H}$. A direct catalytic enantioselective aldol reaction via a novel catalyst design. J Am Chem Soc, 2000, 122: 12003-12004

84 Trost B M, Ito H, Silcoff E R. Asymmetric aldol reaction via a dinuclear zinc catalyst: $\alpha$-hydroxyketones as donors. J Am Chem Soc, 2001, 123: 3367-3368

85 Trost B M, Fettes A, Shireman B T. Direct catalytic asymmetric aldol additions of methyl ynones spontaneous reversal in the rense of enantioinduction. J Am Chem Soc, 2004, 126: 2660-2661

86 Trost B M, Hitce J. Direct asymmetric michael addition to nitroalkenes:vinylogous nucleophilicity under dinuclear zinc catalysis. J Am Chem Soc, 2009, 131: 4572-4573

87 Tamilselvi A, Nethaji M, Mugesh G. Antibiotic resistance: Monoand dinuclear zinc complexes as metallo- $\beta$-lactamase mimics. Chem Eur J, 2006, 12: 7797-7806

88 Molenveld P, Kapsabelis S, Engbersen J F J, et al. Highly efficient phosphate diester transesterification by a calix[4]arene-based dinuclear zinc(II) catalyst. J Am Chem Soc, 1997, 119: 2948-2949

89 Dias E L, Grubbs R H. Synthesis and investigation of homo- and heterobimetallic ruthenium olefin metathesis catalysts exhibiting increased activities. Organometallics, 1998, 17: 2758-2767

90 Sauvage X, Borguet Y, Zaragoza G, et al. Homobimetallic ruthenium vinylidene, allenylidene and indenylidene complexes: Synthesis, characterization and catalytic studies. Adv Synth Catal, 2009, 351: $441-455$
91 Viciano M, Sanau M, Peris E. Ruthenium janus-head complexes with a triazolediylidene ligand structural features and catalytic applications. Organometallics, 2007, 26: 6050-6054

92 Shul'pin G B, Nizova G V, Kozlov Y N, et al. Oxidations by the "hydrogen peroxide-manganese(IV) complex-carboxylic acid" system. Part 4. Efficient acid-base switching between catalase and oxygenase activities of a dinuclear manganese(IV) complex in the reaction with $\mathrm{H}_{2} \mathrm{O}_{2}$ and an alkane. New J Chem, 2002, 26: 1238-1245

93 Woitiski C B, Kozlov Y N, Mandelli D, et al. Oxidations by the system "hydrogen peroxide-dinuclear manganese(IV) complex-carboxylic acid" Part 5. Epoxidation of olefins including natural terpenes. J Mol Catal A Chem, 2004, 222: 103-119

94 Yuri N, Belokon Y N, Caveda C S, et al. The asymmetric addition of trimethylsilyl cyanide to aldehydes catalyzed by chiral (salen) titanium complexes. J Am Chem Soc, 1999, 121: 3968-3973

95 Zhang Z, Wang Z, Zhang R, et al. An efficient titanium catalyst for enantioselective cyanation of aldehydes: cooperative catalysis. Angew Chem Int Ed, 2010, 49: 6746-6750

96 Poyatos M, McNamara W, Incarvito C. A planar chelating bitriazole $\mathrm{N}$-heterocyclic carbene ligand and its rhodium(III) and dirhodium(II) complexes. Chem Commun, 2007: 2267-2269

97 Luo Z, Liu Q, Gong L, et al. The rational design of novel chiral oxovanadium(IV) complexes for highly enantioselective oxidative coupling of 2-naphthols. Chem Commun, 2002: 914-915

98 Luo Z, Liu Q, Gong L. Novel achiral biphenol-derived diastereomeric oxovanadium(IV) complexes for highly enantioselective oxidative coupling of 2-naphthols. Angew Chem Int Ed, 2002, 41: 45324535

99 Yuen H F, Marks T J. Synthesis and catalytic properties of phenylene-bridged binuclear organolanthanide complexes. Organometallics, 2008, 27: 155-158

100 Yuen H F, Marks T J. Phenylene-bridged binuclear organolanthanide complexes as catalysts for intramolecular and intermolecular hydroamination. Organometallics, 2009, 28: 2423-2440

101 Powers D C, Ritter T. Bimetallic Pd(III) complexes in palladiumcatalysed carbon-heteroatom bond formation. Nat Chem, 2009, 1: 302-309

102 Powers D C, Geibel M A L, Klein J E M N, et al. Bimetallic palladium catalysis: Direct observation of Pd(III)-Pd(III) intermediates. J Am Chem Soc, 2009, 131: 17050-17051

103 Powers D C, Benitez D, Tkatchouk E, et al. Bimetallic reductive elimination from dinuclear Pd(III) complexes. J Am Chem Soc, 2010, 132: $14092-14103$

Open Access This article is distributed under the terms of the Creative Commons Attribution License which permits any use, distribution, and reproduction in any medium, provided the original author(s) and source are credited. 